\title{
Gradient observability and sensors for hyperbolic systems
}

A. Boutoulout, H. Bourray and A. Khazari

TSI Team Moulay Ismail University, Faculty of Sciences Meknes Morocco boutouloutali@yahoo.fr

TSI Team Moulay Ismail University, Faculty of Sciences Meknes Morocco hbourrayh@yahoo.fr

TSI Team Moulay Ismail University, Faculty of Sciences Meknes Morocco adil0974@gmail.com

\section{ABSTRACT}

The aim of this paper is to develop useful rigorous results related tothe gradient observability and sensors. The concept of gradient strategic sensors is characterized and applied to the wave equation. This emphasizes the spatial structure and location of the sensors in order that regional gradient observability can be achieved. The developed results are illustrated by many examples, Finally The reconstruct method leads to a numerical algorithm illustrated by simulations.

\section{Indexing terms/Keywords}

Distributed systems, hyperbolic systems, regional gradient observability, sensor strategic.

\section{Academic Discipline And Sub-Disciplines}

E.g., System theory; Control optimal .

\section{SUBJECT CLASSIFICATION}

E.g., Mathematics Subject Classification; 93B07, 93C20.

\section{TYPE (METHOD/APPROACH)}

E.g., Approach HUM (Hilbert Uniqueness Method) ; Approach variational ; Numerical approach.

\section{Council for Innovative Research}

\author{
Peer Review Research Publishing System
}

\section{Journal: INTERNATIONAL JOURNAL OF COMPUTERS \& TECHNOLOGY}

Vol 10, No 2 


\section{INTRODUCTION}

Various reals problems can be formulated within certain concepts of distributed systems analysis. The concepts consist of a set of notions as observability controllability, which enables a better knowledge and understanding of the evolution of the system. The observability concept has been studied at different degrees (exact observability, weakly observability). Systems analysis can be done from a purely theoretical viewpoint (see[1] and [5]). But the study may be also become concrete in some sense, by using the structure of sensors. Recently, the regional observability concept has been developed in Zerrik and El jai (see[2]). It consists in observation of the initial state defined only in a subregion $\omega \subset \Omega$ and then it was extended in Zerrik (see[7] and [10]) to the case where $\omega$ is a part of boundary $\partial \Omega$ of $\Omega$. This concept was extended in Bourray (see[6]) to the regional gradient observability that is interested in observation the system gradient given on a part $\omega$ of $\Omega$. Characterization results of the notion of weak and exact controllability and observability as well

as strategic actuators and sensors, are established (El Jai (see[2] and [4])), Zerrik and al. (see[6] and [9]), Boutoulout (see[8])and Badraoui (see[]). But the almost of these works, the problems are focussed on the systems of parabolic type. The purpose of this paper is to give characterization of the gradient observability for hyperbolic system in connection with sensors structures. More precisely we show that there exists a sensor locations which enable a system regional gradient observable.

The work is organized as follows, in section 2, we introduce the notion of regional weak and exact observability of hyperbolic systems. Then in section 3, we introduce the notion of sensors strategic. Specific properties of this notion

are presented and various situations are also examined. In section 4, we give applications to the wave equation with illustrative examples in one and two dimension.

\section{REGIONAL GRADIENT OBSERVABILITY}

\subsection{Problem statement}

We start by presenting the notations and some preliminary material. Let $\Omega$ be an open bounded subset of $I R^{n}(n=1,2,3)$ with regular boundary $\partial \Omega$ and $\omega$ a subregion of $\Omega$. For $T>0$, we note $\left.Q=\Omega \times\right] 0, T[$, $\Sigma=\partial \Omega \times] 0, T[$ and we consider the following hyperbolic system defined by

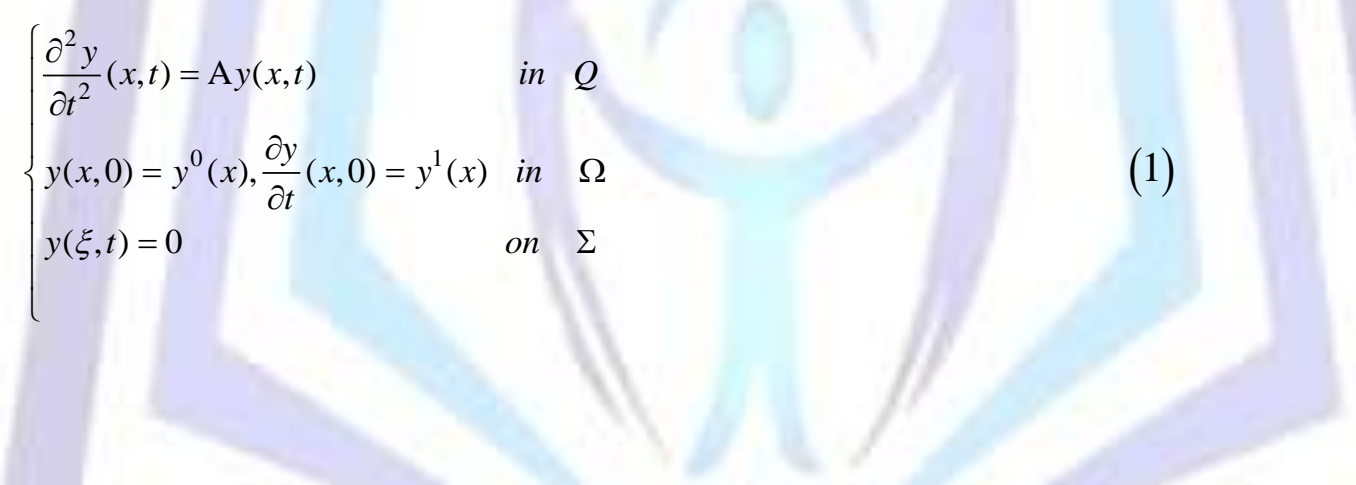

where A is an elliptic and second order operator. The system (1) is observed by measures given by the output function

$$
z(t)=C y(t)
$$

with $C: H_{0}^{1}(\Omega) \rightarrow I R^{q}$ operator linear and depends on the considered sensor structure. Consider the observation space $\mathrm{O}=L^{2}\left(0, T, I R^{q}\right)$ and assume that $\left(y^{0}, y^{1}\right) \in X=H_{0}^{1}(\Omega) \times H_{0}^{1}(\Omega)$.

$$
\bar{y}(t)=\left[\begin{array}{c}
y(t) \\
\frac{\partial y}{\partial t}(t)
\end{array}\right], \quad \bar{y}=\left[\begin{array}{l}
y \\
y
\end{array}\right] \text { and } \overline{\mathrm{A}}=\left[\begin{array}{cc}
0 & I \\
\mathrm{~A} & 0
\end{array}\right]
$$

For $\left(y_{1}, y_{2}\right) \in \mathrm{F}=L^{2}(\Omega) \times L^{2}(\Omega)$ the system (1) is equivalent to

$$
\left\{\begin{array}{l}
\frac{\partial \bar{y}}{\partial t}(t)=\overline{\mathrm{A}} \bar{y}(t) \quad 0<t<T \\
\bar{y}(0)=\bar{y}
\end{array}\right.
$$

augmented with the output function 


$$
\bar{z}(t)=\bar{C} \bar{y}(t)
$$

with $\bar{C}=(C, 0)$ defined by $\bar{C}\left(y^{0}, y^{1}\right)^{T}=\left(C y^{0}, 0\right)^{T}$, the system (3) has a unique solution which can be expressed as $\bar{y}(t)=\bar{S}(t) \bar{y}^{0}$ where $(\bar{S}(t))_{t \geq 0}$ is the semigroup generated by the operator $\overline{\mathrm{A}}$.

Let's consider a basis of eigenfunctions of the operator $\mathrm{A}$, denoted $\Phi_{m_{j}}$, with associated eigenvalues $\lambda_{m}$ with multiplicity $r_{m}$, we can write for $\left(y_{1}, y_{2}\right) \in \mathrm{F}$

$$
\bar{S}(t)\left(\begin{array}{l}
y_{1} \\
y_{2}
\end{array}\right)=\left(\begin{array}{l}
\sum_{m=1}^{\infty} \sum_{j=1}^{r_{m}}\left[\left\langle y_{1}, \Phi_{m_{j}}\right\rangle \cos \left(-\lambda_{m}\right)^{\frac{1}{2}} t+\left(-\lambda_{m}\right)^{-\frac{1}{2}}\left\langle y_{2}, \Phi_{m_{j}}\right\rangle \sin \left(-\lambda_{m}\right)^{\frac{1}{2}} t\right] \Phi_{m_{j}} \\
\sum_{m=1}^{\infty} \sum_{j=1}^{r_{m}}\left[-\left(-\lambda_{m}\right)^{\frac{1}{2}}\left\langle y_{1}, \Phi_{m_{j}}\right\rangle \sin \left(-\lambda_{m}\right)^{\frac{1}{2}} t+\left\langle y_{2}, \Phi_{m_{j}}\right\rangle \cos \left(-\lambda_{m}\right)^{\frac{1}{2}} t\right] \Phi_{m_{j}}
\end{array}\right)
$$

then the output equation can be expressed by

$$
\bar{z}(t)=\bar{C} \bar{S}(t) \bar{y}=\bar{K}(t) \bar{y}, t \in] 0, T[
$$

where $\bar{K}$ be the observation operator defined by

$$
\begin{aligned}
\bar{K}: X & \rightarrow \quad \mathrm{O} \\
\bar{z} & \mapsto \bar{C} \bar{S}(.) \bar{z}
\end{aligned}
$$

which is linear and bounded with the adjoint $\bar{K}^{*}$ given by

$$
\begin{array}{rlc}
\bar{K}^{*}: \mathrm{O} & \rightarrow & X \\
\bar{z}^{*} & \mapsto \int_{0}^{T} \bar{S}^{*}(t) \bar{C}^{*} \bar{z}^{*}(t) d t
\end{array}
$$

Consider the operator $\bar{\nabla}$.given by the formula

$$
\begin{aligned}
\bar{\nabla}: H_{0}^{1}(\Omega) \times H_{0}^{1}(\Omega) & \rightarrow\left(L^{2}(\Omega)\right)^{n} \times\left(L^{2}(\Omega)\right)^{n} \\
\left(y_{1}, y_{2}\right) & \left.\mapsto \bar{\nabla}\left(y_{1}, y_{2}\right)=\left(\nabla y_{1}, \nabla y_{2}\right)\right)
\end{aligned}
$$

where

$$
\begin{aligned}
\nabla: H_{0}^{1}(\Omega) & \rightarrow \quad\left(L^{2}(\Omega)\right)^{n} \\
y & \mapsto \quad \nabla y=\left(\frac{\partial y}{\partial x_{1}}, \ldots, \frac{\partial y}{\partial x_{n}}\right)
\end{aligned}
$$

Its adjoint $\bar{\nabla}^{*}$ is given by

$$
\begin{array}{rlc}
\bar{\nabla}^{*}:\left(L^{2}(\Omega)\right)^{n} \times\left(L^{2}(\Omega)\right)^{n} & \rightarrow & H_{0}^{1}(\Omega) \times H_{0}^{1}(\Omega) \\
\left(y_{1}, y_{2}\right) & \mapsto \bar{\nabla}^{*}\left(y_{1}, y_{2}\right)=\left(\nabla^{*} y_{1}=v_{1}, \nabla^{*} y_{2}=v_{2}\right)
\end{array}
$$

where $v_{i}(i=1,2)$ are the solutions of the following Dirichlet problem

$$
\left\{\begin{array}{ccccc}
\Delta v_{i} & = & -\operatorname{div}\left(y_{i}\right) & \text { in } & \Omega \\
v_{i} & = & 0 & \text { on } & \partial \Omega
\end{array}\right.
$$


The initial state $\bar{y}^{0}$ and its gradient $\bar{\nabla} \bar{y}^{0}$ are assumed to be unknown.

For a nonempty subset $\omega$ of $\Omega$ with positive Lebesgue measure, let $\bar{\chi}_{\omega}$ be the restriction operator defined by

$$
\begin{aligned}
\bar{\chi}_{\omega}:\left(L^{2}(\Omega)\right)^{n} \times\left(L^{2}(\Omega)\right)^{n} & \rightarrow\left(L^{2}(\Omega)\right)^{n} \times\left(L^{2}(\Omega)\right)^{n} \\
\left(y_{1}, y_{2}\right) & \mapsto \bar{\chi}_{\omega}\left(y_{1}, y_{2}\right)=\left(\chi_{\omega} y_{1}, \chi_{\omega} y_{2}\right)
\end{aligned}
$$

where

$$
\begin{aligned}
\chi_{\omega}:\left(L^{2}(\Omega)\right)^{n} & \rightarrow\left(L^{2}(\Omega)\right)^{n} \\
\xi & \mapsto \chi_{\omega} \xi=\left.\xi\right|_{\omega}
\end{aligned}
$$

and

$$
\begin{aligned}
\tilde{\chi}_{\omega}: L^{2}(\Omega) & \rightarrow L^{2}(\omega) \\
\xi & \mapsto \tilde{\chi}_{\omega} \xi=\left.\xi\right|_{\omega}
\end{aligned}
$$

and $\bar{\chi}_{\omega}^{*}$ denotes its adjoint operator, defined as follows

$$
\begin{aligned}
\bar{\chi}_{\omega}^{*}:\left(L^{2}(\omega)\right)^{n} \times\left(L^{2}(\omega)\right)^{n} & \rightarrow\left(L^{2}(\omega)\right)^{n} \times\left(L^{2}(\omega)\right)^{n} \\
\left(y_{1}, y_{2}\right) & \mapsto \bar{\chi}_{\omega}^{*}\left(y_{1}, y_{2}\right)=\left\{\begin{array}{lll}
\left(y_{1}, y_{2}\right) & \text { in } \omega \\
0 & \text { in }(\Omega, \omega)
\end{array}\right.
\end{aligned}
$$

Let us recall that sensors form an important link between a system and its environment. Sensors have an active role and are used to receives the information of the system. This means that the sensors will appear as a right-hind side member of a model, their structure will depend on the shape of the support, the location of the support and the spatial distribution of the measure. A sensor is mathematically de_ned by a pair $(D, f)$, where

1. $D \subset \Omega$ is the support of the sensor.

2. $f$ is the spatial distribution of the measure on the support $D$. In the case of pointwise sensor(internal or boundary), $D$ is reduced to the location $\{b\}$ of the sensor and $f=\delta(., \mathrm{b})$ where $\delta_{b}$ is the Dirac mass concentrated in $b$.

\section{Problem statement}

The system (1) together with the output (2), the problem of gradient observability of the reconstruction gradient of the initial condition in the subregion $\omega \subset \Omega$. This is a natural extension of the observability concept.

Then we introduce the operator $\bar{H}=\bar{\chi}_{\omega} \bar{\nabla} \bar{K}^{*}$ from $\mathrm{O}$ into $\left(L^{2}(\omega)\right)^{n} \times\left(L^{2}(\omega)\right)^{n}$.

Let us recall some de_nitions about the regional observability of the gradient.

\section{Definition 1}

1. The system (1) together with the output (2) is said to be exactly gradient observable in $\omega$ or exactly $G$ observable in $\omega$ if $\quad \operatorname{Im} \bar{H}=\left(L^{2}(\omega)\right)^{n} \times\left(L^{2}(\omega)\right)^{n}$.

2. The system (1) together with the output (2) is said to be weakly gradient observable in $\omega$ or weakly $G$ observable in $\omega$ if $\quad \overline{\operatorname{Im} \bar{H}}=\left(L^{2}(\omega)\right)^{n} \times\left(L^{2}(\omega)\right)^{n}$.

\section{Definition 2}

1. A sensor $(D, f)$ is said to be gradient strategic in $\omega$ if the observed system is weakly $G$-observable in $\omega$.

2. A sequences of sensors $\left(D_{i}, f_{i}\right)_{1 \leq i \leq q}$, is said to be gradient strategic or $G$-strategic if there is one sensor $\left(D_{i_{0}}, f_{i_{0}}\right)$ which is gradient strategic. 


\section{SENSORS GRADIENT STRATEGIC}

Consider the system (1) and assume that the measurements are given by way of sensors $\left(D_{i}, f_{i}\right)_{1 \leq i \leq q}$.

The output equation is then given by:

$$
z(t)=C y\left(t=\left(z_{1}(t), \ldots, z_{1}(q)\right)^{T}\right.
$$

with $D_{i}=\left\{b_{i}\right\}$ and $f=\delta\left(.-b_{i}\right)$ in the pointwise sensor.

and $\quad D_{i} \subset \bar{\Omega}$ with $f \in L^{2}\left(D_{i}\right)$ for the zonal sensor.

We assume that $\left(\Phi_{m_{j}}\right)_{1 \leq j \leq r_{m}, m \geq 1}$ form a complete system in $H_{0}^{1}(\Omega)$, orthonormale in $L^{2}(\Omega)$.

More we assume that $r=\sup r_{m}<\infty$, so we have the following

$$
m
$$

\section{Proposition 3}

The sequence of sensors $\left(D_{i}, f_{i}\right)_{1 \leq i \leq q}$ is $G$-strategic in $\omega$ if and only if

- $q \geq r$

- $\quad \operatorname{rang}\left(G_{m} \gamma_{\omega}^{m}\right)=r_{m}, \forall m \geq 1$

where $\left(G_{m}\right)_{i j}= \begin{cases}\sum_{k=1}^{n}\left\langle\frac{\partial \Phi_{m_{j}}}{\partial x_{k}}, f_{i}\right\rangle_{L^{2}\left(D_{i}\right)} & \text { in the zone case } \\ \sum_{k=1}^{n} \frac{\partial \Phi_{m_{j}}}{\partial x_{k}}\left(b_{i}\right) & \text { in the pointwise case }\end{cases}$

with $1 \leq i \leq q$ and $1 \leq j \leq r_{m}$

$$
\begin{aligned}
& \text { and } \gamma_{\omega}^{m}=\left(\begin{array}{c}
\gamma_{\omega}^{m_{1}} \\
\vdots \\
\gamma_{\omega}^{m_{r_{m}}}
\end{array}\right) \text { with } \gamma_{\omega}^{m_{i}}=\left(\gamma_{\omega}^{m_{i} j_{k}}\right) \quad i=1, \ldots, r_{m_{i}} \text { and } m \geq 1 \\
& \gamma_{\omega}^{m_{i} j_{k}}=\left\langle\Phi_{m_{i}}, \Phi_{j_{k}}\right\rangle L^{2}(\omega) \quad k=1, \ldots, r_{j} \quad \text { and } \quad j \geq 1
\end{aligned}
$$

\section{Proof}

We show that if $\operatorname{rang}\left(G_{m} \gamma_{\omega}^{m}\right)=r_{m}, \forall m \geq 1$, then, the system (1) together with the output (2) is weakly $G$-observable in $\omega$.

We suppose that $\operatorname{Ker} \bar{K} \bar{\nabla}^{*} \bar{\chi}_{\omega}^{*} \neq\{0\}$, then there exists $z^{*}=\left(z^{1^{*}}, z^{2^{*}}\right) \in\left(L^{2}(\omega)\right)^{n} \times\left(L^{2}(\omega)\right)^{n} \quad$ with $\left(z^{1^{*}}, z^{2^{*}}\right) \neq 0$ and $\bar{K} \bar{\nabla}^{*} \bar{\chi}_{\omega}^{*} z^{*}=0$. with

$$
\begin{aligned}
\bar{\chi}_{\omega}^{*} z^{*} & =\left(\chi_{\omega}^{*}{ }^{z^{1 *}}, \chi_{\omega}^{*} z^{2^{*}}\right) \\
& =\left(\tilde{\chi}_{\omega}^{*} z_{1}^{1^{*}}, \ldots ., \tilde{\chi}_{\omega}^{*} z_{n}^{1^{*}}, \tilde{\chi}_{\omega}^{*} z_{1}^{2^{*}}, \ldots ., \tilde{\chi}_{\omega}^{*} z_{n}^{2^{*}}\right)
\end{aligned}
$$

And then 


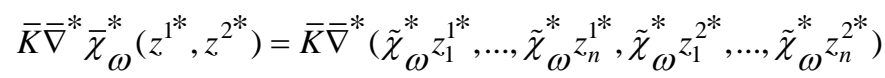

$$
\begin{aligned}
& =\sum_{m=1}^{\infty} \sum_{j=1}^{r_{m}} \sum_{k=1}^{n}\left[\left\langle\tilde{\chi}_{\omega^{*}}^{*} z_{k}^{1^{*}}, \Phi_{m_{j}}\right\rangle \cos \left(-\lambda_{m}\right)^{\frac{1}{2}} t\right. \\
& \left.+\left(-\lambda_{m}\right)^{-\frac{1}{2}}\left\langle\tilde{\chi}_{\omega^{*}}^{*} z_{k}^{2^{*}}, \Phi_{m_{j}}\right\rangle \sin \left(-\lambda_{m}\right)^{\frac{1}{2}} t\right]\left\langle\frac{\partial \Phi_{m_{j}}}{\partial x_{k}}, f_{i}\right\rangle \\
& =0 \quad \forall i=1 \ldots q
\end{aligned}
$$

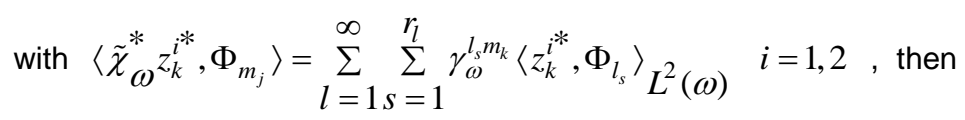

$$
\begin{aligned}
\bar{K} \bar{\nabla}^{*} \bar{\chi}_{\omega}^{*}\left(z^{1^{*}}, z^{2^{*}}\right) & =\sum_{m=1}^{\infty} \sum_{j=1}^{r_{m}} \sum_{k=1}^{n}\left[\sum_{l \geq 1} \sum_{s=1}^{r_{l}} \gamma_{\omega}^{l_{s} m_{k}}\left\langle z_{k}^{1^{*}}, \Phi_{l_{s}}\right\rangle_{L^{2}(\omega)} \cos \left(-\lambda_{m}\right)^{\frac{1}{2}} t\right. \\
& \left.+\left(-\lambda_{m}\right)^{-\frac{1}{2}} \sum_{l=1}^{\infty} \sum_{s=1}^{r_{l}} \gamma_{\omega}^{l_{s} m_{k}}\left\langle z_{k}^{2^{*}}, \Phi_{l_{s}}\right\rangle_{L^{2}(\omega)} \sin \left(-\lambda_{m}\right)^{\frac{1}{2}} t\right]\left\langle\frac{\partial \Phi_{m_{j}}}{\partial x_{k}}, f_{i}\right\rangle \\
& =0 \quad \forall i=1 \ldots q
\end{aligned}
$$

For $T$ large enough the functions $\left\{\sin \left(-\lambda_{n}\right)^{\frac{1}{2}}(.), \cos \left(-\lambda_{n}\right)^{\frac{1}{2}}(.)\right\}_{n \geq 1}$ constitute a complete orthonormale set of $L^{2}(0, T)$, then

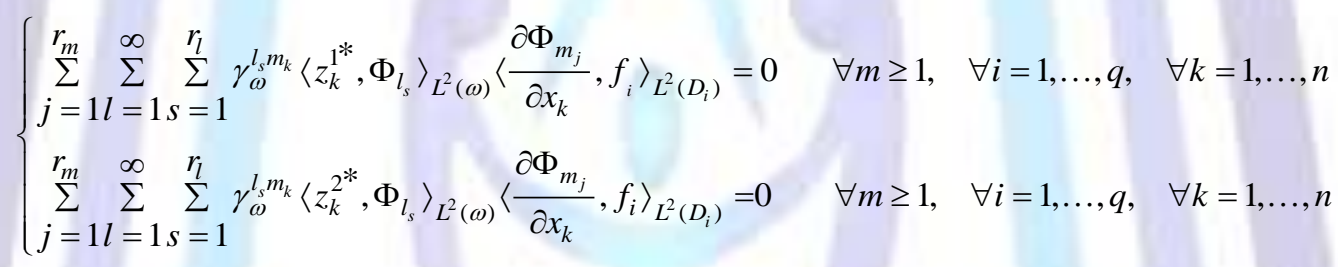

or $z_{k}^{1^{*}}=\sum_{m=1}^{\infty} \sum_{j=1}^{r_{m}} \sum_{k=1}^{\infty} \sum_{s=1}^{r_{l}} \gamma_{\omega}^{l_{s} m_{j}}\left\langle z_{k}^{1^{*}}, \Phi_{l_{s}}\right\rangle_{L^{2}(\omega)} \Phi_{m_{j}} \quad \forall k=1, \ldots, n$

if $z^{1^{*}} \neq 0$ then $\exists k_{0}, \quad 1 \leq k_{0} \leq n, l_{1} \geq 1 \quad$ and $1 \leq s \leq r_{l_{1}}$ with $\left\langle z_{k_{0}}^{1^{*}}, \Phi_{l_{1} s}\right\rangle_{L^{2}(\omega)} \neq 0$

Let's consider :

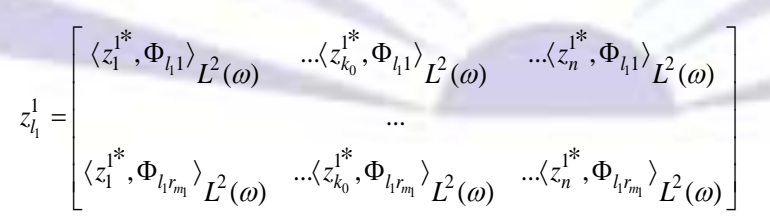

then we obtain $G_{l_{1}} \gamma_{\omega}^{l_{1}} z_{l_{1}}^{1}=0$ or $z_{l_{1}}^{1} \neq 0$, then $\operatorname{rang} G_{l_{1}} \gamma_{\omega}^{l_{1}} \neq r_{l_{1}}$ this is contradiction with $\operatorname{rang} G_{m} \gamma_{\omega}^{m}=r_{m}, \forall m \geq 1$, and the same for $z^{2^{*}} \in\left(L^{2}(\omega)\right)^{n}$ and $z^{2^{*}} \neq 0$.

Finally $\operatorname{Ker} \bar{K} \bar{\nabla}^{*} \bar{\chi}_{\omega}^{*}=\{0\}$, then the system (1) together with the output (2) is weakly $G$-observable in $\omega$.

Conversely, we assume that there exist $l^{\prime} \geq 1$ such that $\operatorname{rang}_{i} \gamma_{\omega}^{i} \neq r_{i}$, then, there exist 


$$
z_{l^{\prime}}=\left[\begin{array}{ccc}
z_{l^{\prime} 1}^{1} & \ldots z_{l^{\prime} 1}^{k_{0}} & \ldots z_{l^{\prime} 1}^{n} \\
& \ldots & \\
z_{l^{\prime} r}^{1} & \ldots z_{l^{\prime} r}^{k_{0}} & \ldots z_{l^{\prime} r}^{n}
\end{array}\right] \text { and } G_{l^{\prime}} \gamma_{\omega}^{l^{\prime}} z_{l^{\prime}}=0
$$

Let's consider $z^{1^{*}} \in\left(L^{2}(\omega)\right)^{n}$ satisfying

$$
\left\{\begin{array}{l}
\left\langle z_{k}^{1^{*}}, \Phi_{l^{\prime} s}\right\rangle L^{2}(\omega)=z_{l^{\prime} s}^{k} \quad \forall s=1 \ldots r_{l^{\prime}}, \forall k=1 \ldots n \\
\left\langle z_{k}^{z^{*}}, \Phi_{l s}\right\rangle L^{2}(\omega)=0 \quad \forall l \neq l^{\prime}, \forall s=1 \ldots r_{l}, \forall k=1 \ldots n
\end{array}\right.
$$

Let's consider $z^{2^{*}} \in\left(L^{2}(\omega)\right)^{n}$ satisfying

$$
\left\{\begin{array}{l}
\left\langle z_{k}^{2^{*}}, \Phi_{l^{\prime} s}\right\rangle L^{2}(\omega)=z_{l^{\prime} s}^{k} \forall s=1 \ldots r_{l^{\prime}}, \forall k=1 \ldots n \\
\left\langle z_{k}^{2^{*}}, \Phi_{l s}\right\rangle L^{2}(\omega)=0 \quad \forall l \neq l^{\prime}, \forall s=1 \ldots r_{l}, \forall k=1 \ldots n
\end{array}\right.
$$

As $G_{l} \cdot \gamma_{\omega}^{l^{\prime}} z_{l^{\prime}}=0$, then we have $z^{*}=\left(z^{1^{*}}, z^{2^{*}}\right) \neq 0$ and $z^{*} \in \operatorname{Ker} \bar{K} \bar{\nabla}^{*} \bar{\chi}_{\omega}^{*}$ (ie) the system (1) together with the output (2) is not weakly $G$-observable in $\omega$.

Finally, the system (1) together with the output (2) is weakly $G$-observable in $\omega$ then $\operatorname{rang}_{m} \gamma_{\omega}^{m}=r_{m} \quad \forall m \geq 1$.

\section{Remark 4}

1. Proposition (3) implies that the required number of sensors is greater than or equal to the largest multiplicity of the eigenvalues.

2. By infinitesimally deforming the domain, the multiplicity of the eigenvalues can be reduced to one (see[3]). Consequently, the regional $G$-observability in $\omega$ can be guarantied using only one sensor.

3. The above result can easily be extended to the case of pointwise sensors(internal or boundary).

\section{APPLICATIONS}

Let us apply the previous results to the case of diffusion systems (1) in one or two dimensions with the output may be pointwise or zone. The system domain will be $\left.\Omega_{1}=\right] 0, a\left[\right.$ in one-dimensional space and $\left.\Omega_{2}=\right] 0, d_{1}[\times] 0, d_{2}[$ in two-dimensional space. The time interval is $] 0, T[$ with $T>0$.

Let $\left.Q_{i}=\Omega_{i} \times\right] 0, T\left[\right.$ and the boundaries $\left.\Sigma_{i}=\partial \Omega_{i} \times\right] 0, T[$.

\subsection{Pointwise sensor}

Let us consider the system with the output may be pointwise sensor $\left(b, \delta_{b}\right)$

$$
\begin{cases}\frac{\partial^{2} y}{\partial t^{2}}(x, t)=\Delta y(x, t) & \text { in } \quad Q_{i} \quad i=1,2 \\ y(x, 0)=y^{0}(x), \frac{\partial y}{\partial t}(x, 0)=y^{1}(x) & \text { in } \quad \Omega_{i} \quad i=1,2 \\ y(\xi, t)=0 & \text { on } \Sigma_{i} \quad i=1,2\end{cases}
$$

\subsubsection{One-dimensional case}

Choose a region $\omega=] \alpha, \beta[\subset] 0, a[$ In this case the eigenvalues and eigenfunctions of the system (6) are given by 


$$
\lambda_{m}=\frac{-m^{2} \pi^{2}}{(\beta-\alpha)^{2}}, \varphi_{m}(x)=\sqrt{\frac{2}{(\beta-\alpha)}} \sin m \pi\left(\frac{x-\alpha}{\beta-\alpha}\right)
$$

the eigenvalues are of multiplicity one. we have

\section{Corollary 5}

The sensor $\left(b, \delta_{b}\right)$ is $G$-strategic in $\omega$ if and only if $\left(\frac{b-\alpha}{\beta-\alpha}\right) \notin S_{G}=\bigcup_{m=1}^{\infty}\left\{\frac{2 k+1}{2 m} \mid 0 \leq k \leq m-\frac{1}{2}\right\}$.

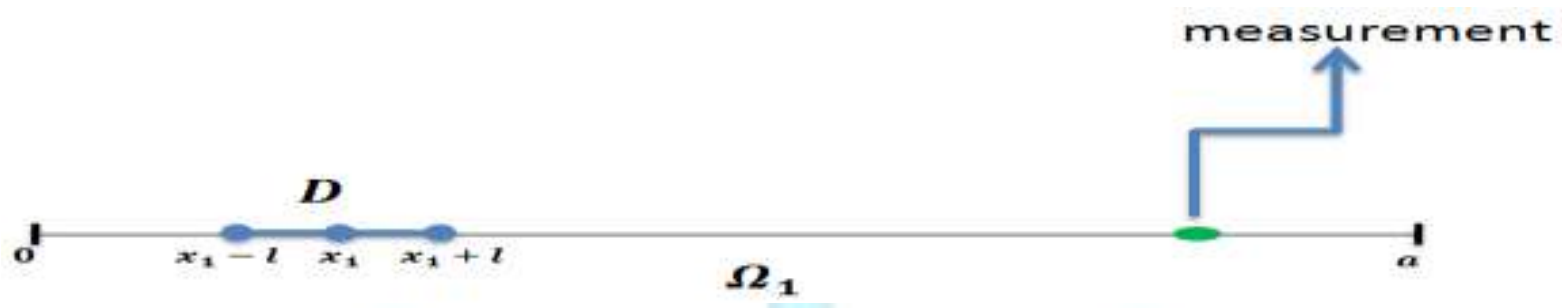

Fig 1: Location of pointwise sensor.

Remark 6

- The system (6) is not weakly observable in $] 0, a\left[\right.$ if and only if $\frac{b}{a} \in S=\bigcup_{m=1}^{\infty}\left\{\frac{k}{m} \mid 1 \leq k<m\right\}$.

- The system (6) is not weakly $G$-observable in ]0, $a$ [ if and only if $\frac{b}{a} \in S_{G}=\bigcup_{m=1}^{\infty}\left\{\frac{2 k+1}{2 m} \mid 0 \leq k \leq m-\frac{1}{2}\right\}$.

We have $S_{G} \subset S$, which shows that there exist sensors which are $G$-strategic without being strategic. +

\subsubsection{Two-dimensional case}

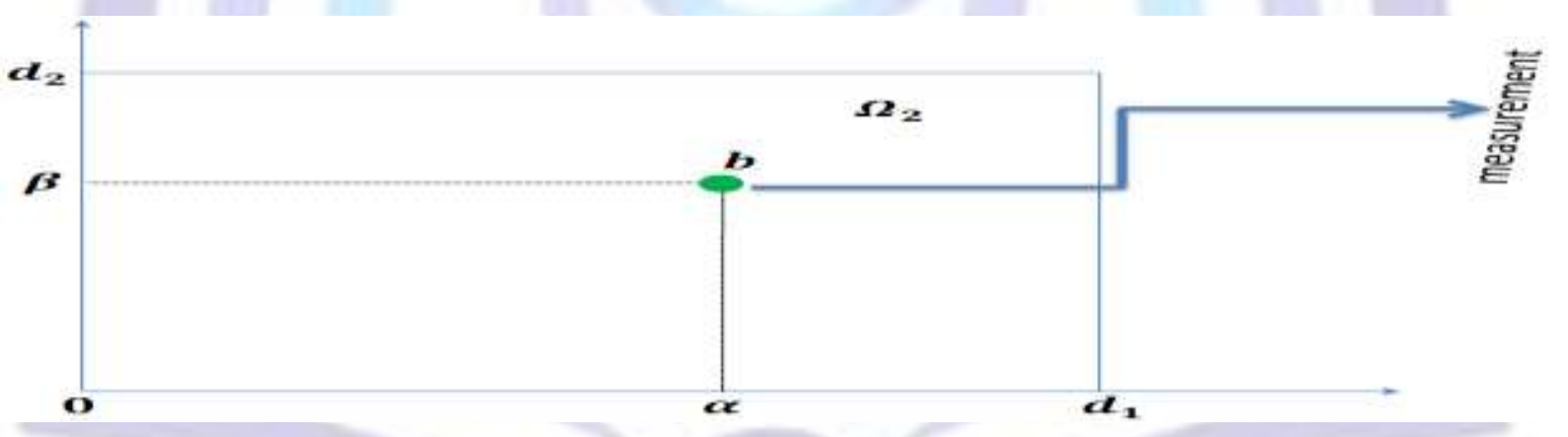

Fig 2: Location of pointwise sensor.

The system (6) is considered by replacing $Q_{1}, \Sigma_{1}$ by $Q_{2}, \Sigma_{2}$. In this case, the eigenvalues and the eigenfunctions for the Dirichlet boundary conditions are given by

$$
\lambda_{m n}=-\left(\frac{m^{2}}{d_{1}^{2}}+\frac{n^{2}}{d_{2}^{2}}\right) \pi^{2}, \varphi_{m n}(x, y)=\frac{2}{\sqrt{d_{1} d_{2}}} \sin \left(\frac{m \pi x}{d_{1}}\right) \sin \left(\frac{n \pi y}{d_{2}}\right)
$$

If $\frac{d_{1}^{2}}{d_{2}^{2}} \notin Q$, the multiplicity of $\lambda_{m n}$ is $r_{m n}=1$ and one sensor may be $G$-strategic.

The output function is given by $z(t)=y(b, t)$, with $b=(\alpha, \beta) \in \Omega$. Then we have the following result.

\section{Corollary 7}


The sensor $\left(b, \delta_{b}\right)$ is not $G$-strategic if there exist $m_{0}, n_{0} \in I N^{*}$ such that

$$
\left(\frac{m_{0}}{d_{1}}+\frac{n_{0}}{d_{2}}\right) \sin \left(\frac{m_{0} \alpha}{d_{1}}+\frac{n_{0} \beta}{d_{2}}\right) \pi=\left(\frac{m_{0}}{d_{1}}-\frac{n_{0}}{d_{2}}\right) \sin \left(\frac{m_{0} \alpha}{d_{1}}-\frac{n_{0} \beta}{d_{2}}\right) \pi
$$

The proof derive immediately from proposition (3).

\subsection{Zone sensor}

In this case we consider the system (6) augmented with the output $z(t)=\int_{D} y(x, t) f(x) d x$ which corresponds to sensor located in the domain $D$.

In the one or two-dimensional cases, the eigenvalues and the eigenfunctions are given by (7) or (8) .

\subsubsection{One-dimensional case}

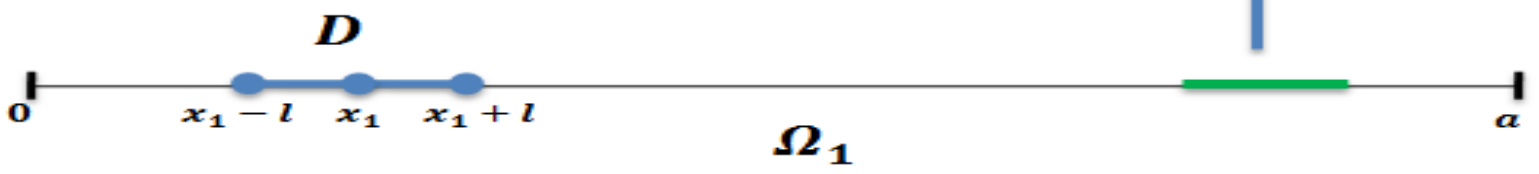

Fig 3 :Location of zone sensor.

Assume that $D=\left[x_{1}-\ell, x_{1}+\ell\right] \subset \Omega$ and $f \in L^{2}(D)$, we have the following result.

\section{Corollary 8}

If $f$ is symmetrical with respect to the line $x=x_{1}$, then the zone sensor $(D, f)$ is not $G$-strategic if one of these conditions is verified

i. $\quad \frac{x_{1}}{a} \in \square$.

ii. There exist $m \in \square^{*}$ such that $\left(\frac{m x_{1}}{a}-\frac{1}{2}\right) \in \square$.

\subsubsection{Two-dimensional case}

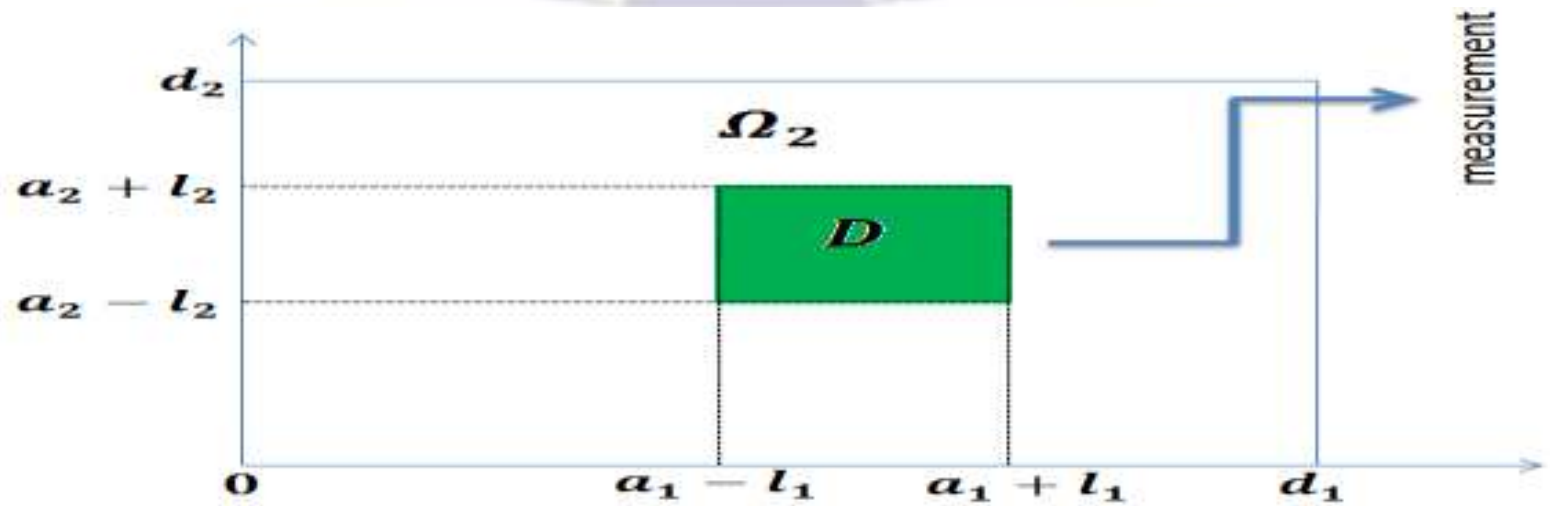

Fig 4: Location of zone sensor. 
We assume that $\frac{d_{1}^{2}}{d_{2}^{2}} \notin \square$ and $r_{m}=1$, then one sensor may be sufficient to ensure the gradient observability. In this case we shall consider the following two situations.

\subsubsection{A rectangular support}

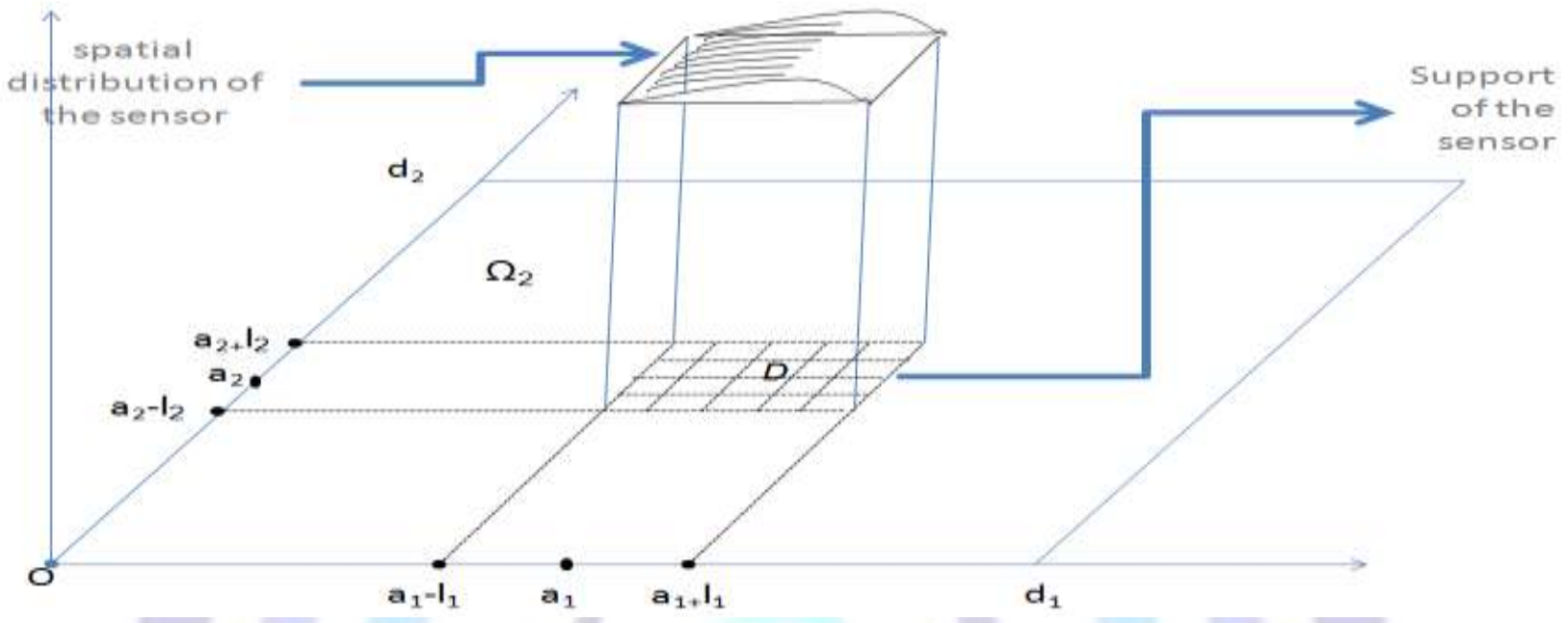

Fig 5: Location of zone sensor with a rectangular support.

Here the sensor support is taken to be $D=\left[a_{1}-\ell_{1}, a_{1}+\ell_{1}\right] \times\left[a_{2}-\ell_{2}, a_{2}+\ell_{2}\right] \subset \Omega_{2}$, we have

\section{Corollary 9}

If $f$ symmetrical with respect to the line $x=a_{1}$ and $y=a_{2}$, then the sensor $(D, f)$ is not $G$-strategic if one of these conditions is verified

i. $\quad \frac{a_{1}}{d_{1}} \in \square, \frac{a_{2}}{d_{2}} \in \square$.

ii. There exist $m_{0}, n_{0} \in \square^{*}$ such that $\left(\frac{m_{0} a_{1}}{d_{1}}-\frac{1}{2}\right) \in \square$ and $\left(\frac{n_{0} a_{2}}{d_{2}}-\frac{1}{2}\right) \in \square$.

\subsubsection{A circular support}

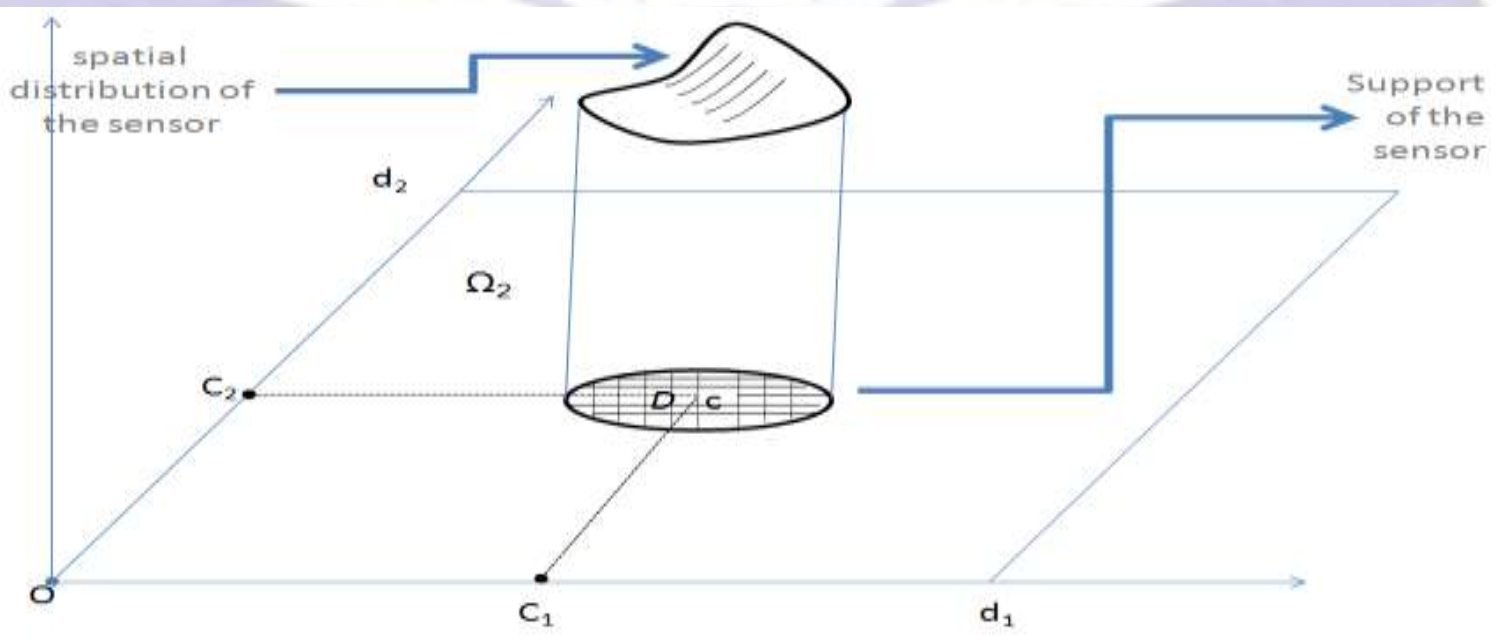

Fig 6: Location of zone sensor with a circular support. 
Let $c=\left(c_{1}, c_{2}\right)$ be the center of the disk $D=D(c, r)$ of radius $r$, then we have

\section{Corollary 10}

If $f$ symmetrical with respect to the line $x=c_{1}$ and $y=c_{2}$, then the sensor $(D, f)$ is not $G$-strategic if one of these conditions is verified

i. $\quad \frac{c_{1}}{d_{1}} \in \square, \frac{c_{2}}{d_{2}} \in \square$

ii. There exist $m_{0}, n_{0} \in \square^{*}$ such that $\left(\frac{m_{0} c_{1}}{d_{1}}-\frac{1}{2}\right) \in \square$ and $\left(\frac{n_{0} c_{2}}{d_{2}}-\frac{1}{2}\right) \in \square$.

The proof of corollary (8); (8) and (10) derive immediately from proposition (3).

This shows that regional gradient observability is linked to location of a disk center and the distribution of measures.

\section{Remark 11}

1. A sensor which is strategic is also G-strategic.

2. We note that the sensor support given above corresponds to real geometry of a sensor in difusion system. The hypothesis of symmetry distribution is physically realistic. For example this would be the case if the sensor was equally distributed over its support $\left(f=\delta \chi_{D}\right)$, where $\chi_{D}$ is the characteristic function and $D \subset \Omega$ is the zone in which the measurements are carried out.

3. From a practical point of view, the distributed system is most often approximated by a finite-dimensional system. Then the conditions of the weakly $G$-observability can be also verified for the finite-dimensional system. For instance, in the pointwise sensor case, if the system is approximated by a three-dimensional system, then the condition of the non $G$-observability is $\frac{\alpha}{d_{1}} \in I_{3}$ where $I_{3}=\{1 / 2,1 / 3,2 / 3\}$ with $\beta=0$.

\section{HILBERT UNIQUENESS METHOD}

In this section, we present an approach which allows the reconstruction of the initial state gradient of (1) in $\omega$. This approach is an extension of the Hilbert Uniqueness Method (H.U.M.) developed by Lions and does not take into account what must be the residual initial gradient in the subregion $\Omega, \omega$.

Let's consider the set

$$
\begin{aligned}
\mathrm{G} & =\left\{\left(h^{1}, h^{2}\right) \in\left(L^{2}(\Omega)\right)^{n} \times\left(L^{2}(\Omega)\right)^{n} \mid h^{1}=h^{2}=0 \quad \text { sur } \quad \Omega, \omega\right\} \\
& \cap\left\{\bar{\nabla}\left(f^{1}, f^{2}\right) \mid\left(f^{1}, f^{2}\right) \in H_{0}^{1}(\Omega) \times H_{0}^{1}(\Omega)\right\}
\end{aligned}
$$

For $\left(\varphi^{0}, \varphi^{1}\right) \in H_{0}^{1}(\Omega) \times H_{0}^{1}(\Omega)$, we consider the following system

$$
\begin{cases}\frac{\partial^{2} \varphi}{\partial t^{2}}(x, t)=\Delta \varphi(x, t) & \text { in } \quad Q \\ \varphi(x, 0)=\varphi^{0}(x), \frac{\partial \varphi}{\partial t}(x, 0)=\varphi^{1}(x) & \text { in } \Omega \\ \varphi(\xi, t)=0 & \text { on } \Sigma\end{cases}
$$

which admits a unique solution $\varphi \in C\left(0, T ; H_{0}^{1}(\Omega)\right) \cap C^{1}\left(0, T ; L^{2}(\Omega)\right)$. (see [5])

We develop our reconstruction approach in the case where the system (1) is observed by pointwise sensors.

Let us define the semi-norm on $\mathrm{G}$ by 


$$
\left(\tilde{\varphi}^{0}, \tilde{\varphi}^{1}\right) \mapsto\left(\tilde{\varphi}^{0}, \tilde{\varphi}^{1}\right) \|_{\mathrm{G}}=\left[\int_{0}^{T}\left(\sum_{k=1}^{n} \frac{\partial \varphi}{\partial x_{k}}(b, t)\right)^{2} d t\right]^{\frac{1}{2}},
$$

We introduce the auxiliary system

$$
\begin{cases}\frac{\partial^{2} \tilde{\psi}}{\partial t^{2}}(x, t)=\Delta \tilde{\psi}(x, t)+\sum_{k=1}^{n} \frac{\partial \varphi}{\partial x_{k}}(b, t) \delta(x-b) & \text { in } \quad Q \\ \tilde{\psi}(x, T)=0, \frac{\partial \tilde{\psi}}{\partial t}(x, T)=0 & \text { in } \Omega \\ \frac{\partial \tilde{\psi}}{\partial v}(\xi, t)=0 & \text { on } \Sigma\end{cases}
$$

The solution $\tilde{\psi}$ of $(11)$ is in $C\left(0, T ; H_{0}^{1}(\Omega)\right) \cap C^{1}\left(0, T ; L^{2}(\Omega)\right)$. (see [8]).

The resolution of the system (11) provides $\tilde{\psi}(x, 0)=\tilde{\psi}^{0}(x)$ and $\frac{\partial \tilde{\psi}}{\partial t}(x, 0)=\tilde{\psi}^{1}(x)$.

When the semi norm (10) is a norm, we also denote by $G$ the completion of $G$ and we consider the operator

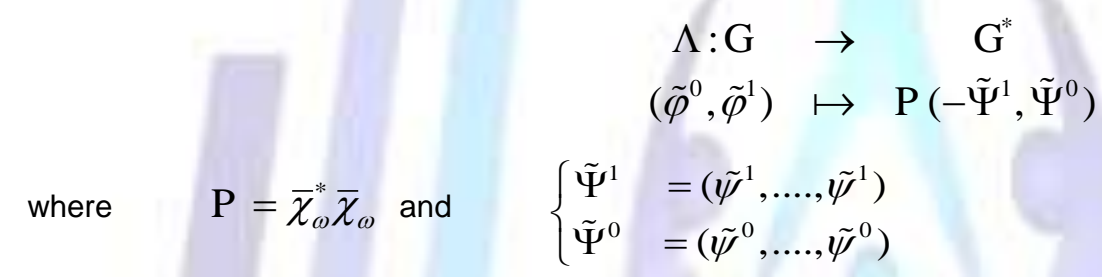

With $\tilde{\Psi}(x, 0)=\tilde{\Psi}^{0}(x)$ and $\frac{\partial \tilde{\Psi}}{\partial t}(x, 0)=\tilde{\Psi}^{1}(x)$

We introduce the system

$$
\begin{cases}\frac{\partial^{2} \bar{\psi}}{\partial t^{2}}(x, t)=\Delta \bar{\psi}(x, t)+\sum_{k=1}^{n} \frac{\partial y}{\partial x_{k}}(b, t) \delta(x-b) & \text { in } Q \\ \bar{\psi}(x, T)=0, \frac{\partial \bar{\psi}}{\partial t}(x, T)=0 & \text { in } \Omega \\ \frac{\partial \bar{\psi}}{\partial v}(\xi, t)=0 & \text { on } \Sigma\end{cases}
$$

If $\left(\tilde{\varphi}^{0}, \tilde{\varphi}^{1}\right)$ is chosen such that $\tilde{\psi}^{1}=\bar{\psi}^{1}$ and $\tilde{\psi}^{0}=\bar{\psi}^{0}$ in $\omega$, then the system (12) looks like the adjoint of the system (1), and the regional gradient observability amounts to the conditions for solving the equation

$$
\Lambda\left(\tilde{\varphi}^{0}, \tilde{\varphi}^{1}\right)=\mathrm{P}\left(-\bar{\Psi}^{1}, \bar{\Psi}^{0}\right)
$$

where $\left\{\begin{aligned} \bar{\Psi}^{1} & =\left(\frac{\partial \bar{\psi}}{\partial t}, \ldots ., \frac{\partial \bar{\psi}}{\partial t}\right) \text { with } \bar{\psi} \quad \text { being the solution of (12) } \\ \bar{\Psi}^{0} & =(\bar{\psi}, \ldots ., \bar{\psi})\end{aligned}\right.$

\section{Remark 12}

Among choice of $\tilde{\varphi}^{0}$ and $\tilde{\varphi}^{1}$ who realizes $\tilde{\psi}^{0}=\bar{\psi}^{0}$ and $\tilde{\psi}^{1}=\bar{\psi}^{1}$ in $\omega$.

For $\tilde{\psi}^{0}=y_{1}^{\prime 0}$ and $\tilde{\psi}^{1}=y_{1}^{\prime 1}$, this choice is not unique but if we show that the operator $\Lambda$ is an isomorphism then (13) admit a unique solution $\left(\tilde{\varphi}^{0}, \tilde{\varphi}^{1}\right)$ which will coincide with $\left(y_{1}^{\prime 0}, y_{1}^{11}\right)$ in $\omega$. 
We consider the decomposition of the initial state as

$$
y^{0}=\left\{\begin{array}{ccc}
y_{1}^{0} & \text { in } & \omega \\
y_{2}^{0} & \text { in } & \Omega \backslash \omega
\end{array} \quad \text { and } \quad y^{1}=\left\{\begin{array}{ccc}
y_{1}^{1} & \text { in } & \omega \\
y_{2}^{1} & \text { in } & \Omega \backslash \omega
\end{array}\right.\right.
$$

and the decomposition of the gradient of the initial state as follows

$$
\nabla y^{0}=\left\{\begin{array}{ccc}
y_{1}^{\prime 0} & \text { in } & \omega \\
y_{2}^{\prime 0} & \text { in } & \Omega \backslash \omega
\end{array} \quad \text { and } \quad \nabla y^{1}=\left\{\begin{array}{ccc}
y_{1}^{\prime 1} & \text { in } & \omega \\
y_{2}^{\prime 1} & \text { in } & \Omega \backslash \omega
\end{array}\right.\right.
$$

\section{Proposition 13}

If the sensor $\left(b, \delta_{b}\right)$ is $G$-strategic in $\omega$, then the semi norm (10) becomes a norm and the equation (13) has a unique solution $\left(\tilde{\varphi}^{0}, \tilde{\varphi}^{1}\right)$ which corresponds to $\left(y_{1}{ }^{0}, y_{1}^{1}\right)$ the gradient of the initial state to be observed in the subregion $\omega$.

\section{Proof}

If the system (1) together with the output (2) is weakly $G$-observable in $\omega$, then (10) defines a norm in $G$ Let's consider $\left(\Phi_{m}\right)$ the eigenfunctions of the operator $\Delta$, without loss of generality, we assume that the eigenvalues $\lambda_{m}$ are simple (see[3]).

Let's $\left(\tilde{\varphi}^{0}, \tilde{\varphi}^{1}\right) \in \mathrm{G}$ such as $\mid\left(\tilde{\varphi}^{0}, \tilde{\varphi}^{1}\right) \|_{\mathrm{G}}=0$, we show that $\left(\tilde{\varphi}^{0}, \tilde{\varphi}^{1}\right)=(0,0) ; \mathrm{I}\left(\tilde{\varphi}^{0}, \tilde{\varphi}^{1}\right) \|_{\mathrm{G}}=0$ gives

$$
\sum_{k=1}^{n} \sum_{i=1}^{\infty}\left[\left\langle\varphi^{0}, \Phi_{i}\right\rangle_{L^{2}(\Omega)} \cos \left(-\lambda_{i}\right)^{\frac{1}{2}} t+\left(-\lambda_{i}\right)^{-\frac{1}{2}}\left\langle\varphi^{1}, \Phi_{i}\right\rangle_{L^{2}(\Omega)} \sin \left(-\lambda_{i}\right)^{\frac{1}{2}} t\right] \frac{\partial \Phi_{i}}{\partial x_{k}}(b)=0
$$

for $T$ large enough the functions $\left\{\left(\sin \left(-\lambda_{i}\right)^{\frac{1}{2}} t\right)_{n \geq 1},\left(\cos \left(-\lambda_{i}\right)^{\frac{1}{2}} t\right)_{n \geq 1}\right\}$ form a complete orthonormal set in $L^{2}(0, T)$, we obtain

$$
\left\langle\varphi^{0}, \Phi_{i}\right\rangle_{L^{2}(\Omega)} \sum_{k=1}^{n} \frac{\partial \Phi_{i}}{\partial x_{k}}(b)=0 \quad \text { and } \quad\left\langle\varphi^{1}, \Phi_{i}\right\rangle_{L^{2}(\Omega)} \sum_{k=1}^{n} \frac{\partial \Phi_{i}}{\partial x_{k}}(b)=0 \quad \forall i \geq 1
$$

or the sensor $\left(b, \delta_{b}\right)$ is $G$-strategic, then $\sum_{k=1}^{n} \frac{\partial \Phi_{i}}{\partial x_{k}}(b) \neq 0 \quad \forall i \geq 1$ then $\left\langle\varphi^{0}, \Phi_{i}\right\rangle=\left\langle\varphi^{1}, \Phi_{i}\right\rangle=0 \quad \forall i \geq 1$ which implies $\left(\varphi^{0}, \varphi^{1}\right)=(0,0)$ then $\left(\tilde{\varphi}^{0}, \tilde{\varphi}^{1}\right)=(0,0)$

We show that $\Lambda$ is an isomorphism

Multiplying (11) by $\frac{\partial \varphi}{\partial x_{k}}$ and integrating over $Q$, we obtain

$$
\begin{aligned}
\int_{0}^{T}\left\langle\frac{\partial \varphi}{\partial x_{k}}(x, t), \frac{\partial^{2} \tilde{\psi}}{\partial t^{2}}(x, t)\right\rangle_{L^{2}(\Omega)} d t & =\int_{0}^{T}\left\langle\frac{\partial \varphi}{\partial x_{k}}(x, t), \Delta \tilde{\psi}(x, t)\right\rangle_{L^{2}(\Omega)} d t \\
& +\int_{0}^{T}\left\langle\frac{\partial \varphi}{\partial x_{k}}(x, t), \sum_{l=1}^{n} \frac{\partial \varphi}{\partial x_{l}}(\mathrm{~b}, t) \delta(x-b)\right\rangle_{L^{2}(\Omega)}
\end{aligned}
$$

which gives 


$$
\begin{aligned}
{\left.\left[\frac{\partial \varphi}{\partial x_{k}}(x, t), \frac{\partial \tilde{\psi}}{\partial t}(x, t)\right\rangle_{L^{2}(\Omega)}\right]_{0}^{T} } & -\left[\left\langle\frac{\partial}{x_{k}}\left(\frac{\partial \varphi}{\partial t}(x, t)\right), \tilde{\psi}(x, t)\right\rangle_{L^{2}(\Omega)}\right]_{0}^{T} \\
& +\int_{0}^{T}\left\langle\frac{\partial}{\partial x_{k}} \frac{\partial^{2} \varphi}{\partial t^{2}}(x, t), \tilde{\psi}(x, t)\right\rangle_{L^{2}(\Omega)} d t \\
& =\int_{0}^{T}\left\langle\frac{\partial \varphi}{\partial x_{k}}(x, t), \Delta \tilde{\psi}(x, t)\right\rangle_{L^{2}(\Omega)} d t \\
& +\int_{0}^{T} \frac{\partial \varphi}{\partial x_{k}}(b, t) \sum_{l=1}^{n} \frac{\partial \varphi}{\partial x_{l}}(b, t) d t
\end{aligned}
$$

with the final condition, we obtain

$$
\begin{aligned}
-\left\langle\frac{\partial \varphi}{\partial x_{k}}(x, 0), \frac{\partial \tilde{\psi}}{\partial t}(x, 0)\right\rangle_{L^{2}(\Omega)} & +\left\langle\frac{\partial}{\partial x_{k}} \frac{\partial \varphi}{\partial t}(x, 0), \tilde{\psi}(x, 0)\right\rangle_{L^{2}(\Omega)} \\
& +\left\langle\Delta \frac{\partial \varphi}{\partial x_{k}}(x, t), \tilde{\psi}(x, t)\right\rangle_{L^{2}(Q)} \\
& =\left\langle\frac{\partial \varphi}{\partial x_{k}}(x, t), \Delta \tilde{\psi}(x, t)\right\rangle_{L^{2}(Q)} \\
& +\int_{0}^{T} \frac{\partial \varphi}{\partial x_{k}}(b, t) \sum_{l=1}^{n} \frac{\partial \varphi}{\partial x_{l}}(b, t) d t
\end{aligned}
$$

Using the Green formula, we obtain

$$
\begin{aligned}
-\left\langle\frac{\partial \varphi}{\partial x_{k}}(x, 0), \frac{\partial \tilde{\psi}}{\partial t}(x, 0)\right\rangle_{L^{2}(\Omega)} & +\left\langle\frac{\partial}{\partial x_{k}} \frac{\partial \varphi}{\partial t}(x, 0), \tilde{\psi}(x, 0)\right\rangle_{L^{2}(\Omega)} \\
& =\int_{0}^{T} \frac{\partial \varphi}{\partial x_{k}}(b, t) \sum_{l=1}^{n} \frac{\partial \varphi}{\partial x_{l}}(b, t) d t
\end{aligned}
$$

and then

$$
\left\langle\left(-\frac{\partial \tilde{\psi}}{\partial x_{k}}(x, 0), \tilde{\psi}(x, 0)\right),\left(\frac{\partial \varphi}{\partial x_{k}}(x, 0), \frac{\partial}{\partial t} \frac{\partial \varphi}{\partial x_{k}}(x, 0)\right)\right\rangle_{L^{2}(\Omega)}=\int_{0}^{T} \frac{\partial \varphi}{\partial x_{k}}(b, t) \sum_{l=1}^{n} \frac{\partial \varphi}{\partial x_{l}}(b, t) d t
$$

Thus

$$
\sum_{k=1}^{n}\left\langle\left(-\frac{\partial \tilde{\psi}}{\partial x_{k}}(x, 0), \tilde{\psi}(x, 0)\right),\left(\frac{\partial \varphi}{\partial x_{k}}(x, 0), \frac{\partial}{\partial t} \frac{\partial \varphi}{\partial x_{k}}(x, 0)\right)\right\rangle_{L^{2}(\Omega)}=\sum_{k=1}^{n} \int_{0}^{T} \frac{\partial \varphi}{\partial x_{k}}(b, t) \sum_{l=1}^{n} \frac{\partial \varphi}{\partial x_{l}}(b, t) d t
$$

Finally

$$
\begin{aligned}
\left\langle\Lambda\left(\tilde{\varphi}^{0}, \tilde{\varphi}^{1}\right),\left(\tilde{\varphi}^{0}, \tilde{\varphi}^{1}\right)\right\rangle= & \int_{0}^{T}\left(\sum_{l=1}^{n} \frac{\partial \varphi}{\partial x_{l}}(b, t)\right)^{2} d t \\
& \#\left(\tilde{\varphi}^{0}, \tilde{\varphi}^{1}\right) \|_{\mathrm{G}}^{2}, \quad \forall\left(\tilde{\varphi}^{0}, \tilde{\varphi}^{1}\right) \in \mathrm{G}
\end{aligned}
$$

which proves that $\Lambda$ is an isomorphism and (13) has a unique solution which corresponds to the gradient of the initial condition to be estimated in the subregion $\omega$.

\section{ESTIMATION OF THE INITIAL CONDITIONS}


In this section we show how one can solve the equation (13) and we give explicit expressions for $y_{1}^{\prime 0}$ and $y_{1}^{\prime 1}$ in $\omega$. which can be used for numerical simulations as shown in the next section. Using standard optimization techniques, it is well known that solving (13) is equivalent to the minimization problem

$$
\begin{aligned}
\mathrm{R}\left(\tilde{\varphi}^{0}, \tilde{\varphi}^{1}\right) & =\frac{1}{2}\left\langle\Lambda\left(\tilde{\varphi}^{0}, \tilde{\varphi}^{1}\right),\left(\tilde{\varphi}^{0}, \tilde{\varphi}^{1}\right)\right\rangle-\left\langle\mathrm{P}\left(-\bar{\Psi}^{\prime}(0), \bar{\Psi}(0)\right),\left(\tilde{\varphi}^{0}, \tilde{\varphi}^{1}\right)\right\rangle \\
& =\frac{1}{2} \int_{0}^{T}\left(\sum_{l=1}^{n} \frac{\partial \varphi}{\partial x_{l}}(b, t)\right)^{2} d t+\left\langle\bar{\Psi}^{1}, \tilde{\varphi}^{0}\right\rangle-\left\langle\bar{\Psi}^{0}, \tilde{\varphi}^{1}\right\rangle
\end{aligned}
$$

with $\quad \mathrm{P}\left(\bar{\Psi}^{\prime}(0), \bar{\Psi}(0)\right)=\left\langle\bar{\Psi}^{1}, \bar{\Psi}^{0}\right\rangle$

From $\varphi(b, t)=\sum_{m=1}^{\infty}\left[\left\langle\varphi^{0}, \Phi_{m}\right\rangle \cos \sqrt{-\lambda_{m}} t+\frac{\left\langle\varphi^{1}, \Phi_{m}\right\rangle}{\sqrt{-\lambda_{m}}} \sin \sqrt{-\lambda_{m}} t\right] \Phi_{m}(b)$

We have $\sum_{l=1}^{n} \frac{\partial \varphi}{\partial x_{l}}(b, t)=\sum_{l=1}^{n} \sum_{m=1}^{\infty}\left[\left\langle\varphi^{0}, \Phi_{m}\right\rangle \cos \sqrt{-\lambda_{m}} t+\frac{\left\langle\varphi^{1}, \Phi_{m}\right\rangle}{\sqrt{-\lambda_{m}}} \sin \sqrt{-\lambda_{m}} t\right] \frac{\partial \Phi_{m}}{\partial x_{l}}(b)$

Then

$$
\int_{0}^{T}\left(\sum_{l=1}^{n} \frac{\partial \varphi}{\partial x_{l}}(b, t)\right)^{2} d t=\int_{0}^{T}\left[\sum_{l=1}^{n} \sum_{m=1}^{\infty}\left(\left\langle\varphi^{0}, \Phi_{m}\right\rangle \cos \sqrt{-\lambda_{m}} t+\frac{\left\langle\varphi^{1}, \Phi_{m}\right\rangle}{\sqrt{-\lambda_{m}}} \sin \sqrt{-\lambda_{m}} t\right) \frac{\partial \Phi_{m}}{\partial x_{l}}(b)\right]^{2} d t
$$

we obtain

$$
\lim _{T \rightarrow+\infty} \frac{1}{2 T} \int_{0}^{T}\left(\sum_{l=1}^{n} \frac{\partial \varphi}{\partial x_{l}}(b, t)\right)^{2} d t=\frac{1}{4} \sum_{m=1}^{\infty}\left[\left\langle\varphi^{0}, \Phi_{m}\right\rangle^{2}-\frac{1}{\lambda_{m}}\left\langle\varphi^{1}, \Phi_{m}\right\rangle^{2}\right]\left(\sum_{l=1}^{n} \frac{\partial \Phi_{m}}{\partial x_{l}}(b)\right)^{2}
$$

For $T$ large enough we have

$$
\frac{1}{2} \int_{0}^{T}\left(\sum_{l=1}^{n} \frac{\partial \varphi}{\partial x_{l}}(b, t)\right)^{2} d t \square \frac{T}{4} \sum_{m=1}^{\infty}\left[\left\langle\varphi^{0}, \Phi_{m}\right\rangle^{2}-\frac{1}{\lambda_{m}}\left\langle\varphi^{1}, \Phi_{m}\right\rangle^{2}\right]\left(\sum_{l=1}^{n} \frac{\partial \Phi_{m}}{\partial x_{l}}(b)\right)^{2}
$$

But $\quad \varphi^{0}(x)=\sum_{m=1}^{\infty}\left\langle\varphi^{0}, \Phi_{m}\right\rangle \Phi_{m}(x) \quad$ and $\quad \varphi^{1}(x)=\sum_{m=1}^{\infty}\left\langle\varphi^{1}, \Phi_{m}\right\rangle \Phi_{m}(x)$

Then $\quad \tilde{\varphi}^{0}(x)=\sum_{m=1}^{\infty}\left\langle\varphi^{0}, \Phi_{m}\right\rangle \nabla \Phi_{m}(x) \quad$ and $\quad \tilde{\varphi}^{1}(x)=\sum_{m=1}^{\infty}\left\langle\varphi^{1}, \Phi_{m}\right\rangle \nabla \Phi_{m}(x)$

Finally

$$
\left\{\begin{array}{l}
\left\langle\bar{\Psi}^{1}, \tilde{\varphi}^{0}\right\rangle=\sum_{l=1}^{n} \sum_{m=1}^{\infty}\left\langle\varphi^{0}, \Phi_{m}\right\rangle\left\langle\bar{\psi}^{1}, \frac{\partial \Phi_{m}}{\partial x_{l}}\right\rangle \\
\left\langle\bar{\Psi}^{0}, \tilde{\varphi}^{1}\right\rangle=\sum_{l=1}^{n} \sum_{m=1}^{\infty}\left\langle\varphi^{1}, \Phi_{m}\right\rangle\left\langle\bar{\psi}^{0}, \frac{\partial \Phi_{m}}{\partial x_{l}}\right\rangle
\end{array}\right.
$$

the minimization of $(16)$ is equivalent to find

$$
\begin{aligned}
\inf _{\left(\varphi^{0}, \varphi^{1}\right)} \frac{T}{4} \sum_{m=1}^{\infty}\left[\left\langle\varphi^{0}, \Phi_{m}\right\rangle^{2}-\frac{1}{\lambda_{m}}\left\langle\varphi^{1}, \Phi_{m}\right\rangle^{2}\right]\left(\sum_{l=1}^{n} \frac{\partial \Phi_{m}}{\partial x_{l}}(b)\right)^{2} \\
+\sum_{m=1}^{\infty}\left(\left\langle\varphi^{0}, \Phi_{m}\right\rangle \sum_{l=1}^{n}\left\langle\bar{\psi}^{1}, \frac{\partial \Phi_{m}}{\partial x_{l}}\right\rangle-\left\langle\varphi^{1}, \Phi_{m}\right\rangle \sum_{l=1}^{n}\left\langle\bar{\psi}^{0}, \frac{\partial \Phi_{m}}{\partial x_{l}}\right\rangle\right)
\end{aligned}
$$

with separation of the variables we obtain 


$$
\left\{\begin{array}{l}
\inf _{\varphi^{0}} \sum_{m=1}^{\infty}\left[\frac{T}{4}\left\langle\varphi^{0}, \Phi_{m}\right\rangle^{2}\left(\sum_{l=1}^{n} \frac{\partial \Phi_{m}}{\partial x_{l}}(b)\right)^{2}+\left\langle\varphi^{0}, \Phi_{m}\right\rangle \sum_{l=1}^{n}\left\langle\bar{\psi}^{1}, \frac{\partial \Phi_{m}}{\partial x_{l}}\right\rangle\right] \\
\inf _{\varphi^{1}} \sum_{m=1}^{\infty}\left[\frac{-T}{4} \frac{1}{\lambda_{m}}\left\langle\varphi^{1}, \Phi_{m}\right\rangle^{2}\left(\sum_{l=1}^{n} \frac{\partial \Phi_{m}}{\partial x_{l}}(b)\right)^{2}-\left\langle\varphi^{1}, \Phi_{m}\right\rangle \sum_{l=1}^{n}\left\langle\bar{\psi}^{0}, \frac{\partial \Phi_{m}}{\partial x_{l}}\right\rangle\right]
\end{array}\right.
$$

we have $\quad \frac{\partial}{\partial \varphi^{0}}\left[\frac{T}{4}\left\langle\varphi^{0}, \Phi_{m}\right\rangle^{2}\left(\sum_{l=1}^{n} \frac{\partial \Phi_{m}}{\partial x_{l}}(b)\right)^{2}+\left\langle\varphi^{0}, \Phi_{m}\right\rangle \sum_{l=1}^{n}\left\langle\bar{\psi}^{1}, \frac{\partial \Phi_{m}}{\partial x_{l}}\right\rangle\right]=0 \quad \forall m \geq 1$

which is equivalent to

$$
\left\langle\varphi^{0}, \Phi_{m}\right\rangle=-\frac{2}{T} \frac{\left\langle\bar{\Psi}^{1}, \nabla \Phi_{m}\right\rangle}{\left(\sum_{l=1}^{n} \frac{\partial \Phi_{m}}{\partial x_{l}}(b)\right)^{2}} \quad \forall m \geq 1
$$

Idem for

$$
\left\langle\varphi^{1}, \Phi_{m}\right\rangle=-\frac{2 \lambda_{m}}{T} \frac{\left\langle\bar{\Psi}^{0}, \nabla \Phi_{m}\right\rangle}{\left(\sum_{l=1}^{n} \frac{\partial \Phi_{m}}{\partial x_{l}}(b)\right)^{2}} \quad \forall m \geq 1
$$

and it is known that $\bar{\psi}(x, t)=\sum_{k=1}^{\infty} \bar{\psi}_{k}(t) \Phi_{k}(x)$ with $\quad \bar{\psi}_{k}(t)=\frac{\Phi_{k}(b)}{\sqrt{-\lambda_{k}}} \int_{0}^{T} \sum_{i=1}^{n} \frac{\partial y}{\partial x_{i}}(b, s) \sin \sqrt{-\lambda_{k}}(s-t) d s$ but $\quad \bar{\psi}(x, 0)=\bar{\psi}^{0}(x)=\sum_{k=1}^{\infty}\left\langle\bar{\psi}^{0}, \Phi_{k}\right\rangle \Phi_{k}(x)$ and $\frac{\partial \bar{\psi}}{\partial t}(x, 0)=\bar{\psi}^{1}(x)=\sum_{k=1}^{\infty}\left\langle\bar{\psi}^{1}, \Phi_{k}\right\rangle \Phi_{k}(x)$ then $\quad \bar{\psi}^{0}=\sum_{k=1}^{\infty} \frac{\Phi_{k}(b)}{\sqrt{-\lambda_{k}}} \int_{0}^{T} \sum_{i=1}^{n} \frac{\partial y}{\partial x_{i}}(b, s) \sin \sqrt{-\lambda_{k}} s d s \Phi_{k}(x)$ and $\quad \bar{\psi}^{1}=\sum_{k=1}^{\infty}-\Phi_{k}(b) \int_{0}^{T} \sum_{i=1}^{n} \frac{\partial y}{\partial x_{i}}(b, s) \cos \sqrt{-\lambda_{k}} s d s \Phi_{k}(x)$ finally $\left\langle\bar{\Psi}^{0}, \nabla \Phi_{m}\right\rangle=\sum_{l=1}^{n} \sum_{k=1}^{\infty} \frac{\Phi_{k}(b)}{\sqrt{-\lambda_{k}}} \int_{0}^{T} \sum_{i=1}^{n} \frac{\partial y}{\partial x_{i}}(b, s) \sin \sqrt{-\lambda_{k}} s d s\left\langle\Phi_{k}, \frac{\partial \Phi_{m}}{\partial x_{l}}\right\rangle_{L^{2}(\omega)}$ and $\quad\left\langle\bar{\Psi}^{1}, \nabla \Phi_{m}\right\rangle=\sum_{l=1}^{n} \sum_{k=1}^{\infty}-\Phi_{k}(b) \int_{0}^{T} \sum_{i=1}^{n} \frac{\partial y}{\partial x_{i}}(b, s) \cos \sqrt{-\lambda_{k}} s d s\left\langle\Phi_{k}, \frac{\partial \Phi_{m}}{\partial x_{l}}\right\rangle_{L^{2}(\omega)}$ Finally we obtain the explicit formulae for the initial gradient $y_{1}^{\prime 0}$ and $y_{1}^{1}$ :

$$
y_{1}^{\prime 0}(x)=\left\{-\frac{2}{T} \sum_{m=1}^{\infty}\left[\frac{\sum_{l=1 k=1}^{n} \sum_{k}^{\infty}-\Phi_{k}(b) \int_{0}^{T} \sum_{i=1}^{n} \frac{\partial y}{\partial x_{i}}(b, s) \cos \sqrt{-\lambda_{k}} s d s\left\langle\Phi_{k}, \frac{\partial \Phi_{m}}{\partial x_{l}}\right\rangle_{L^{2}(\omega)}}{\left(\sum_{l=1}^{n} \frac{\partial \Phi_{m}}{\partial x_{l}}(b)\right)^{2}}\right] \nabla \Phi_{m}(x) \quad x \in \omega\right.
$$


And

$$
y_{1}^{\prime 1}(x)=\left\{\begin{array}{cc}
-\frac{2}{T} \sum_{m=1}^{\infty}\left[\lambda_{m} \frac{\sum_{l=1}^{n} \sum_{k=1}^{\infty} \frac{\Phi_{k}(b)}{\sqrt{-\lambda_{k}} \int_{0}^{T} \sum_{i=1}^{n} \frac{\partial y}{\partial x_{i}}(b, s) \sin \sqrt{-\lambda_{k}} s d s\left\langle\Phi_{k}, \frac{\partial \Phi_{m}}{\partial x_{l}}\right\rangle_{L^{2}(\omega)}}}{\left(\sum_{l=1}^{n} \frac{\partial \Phi_{m}}{\partial x_{l}}(b)\right)^{2}}\right. \\
0
\end{array}\right] \nabla \Phi_{m}(x) \quad x \in \omega
$$

These formulae are of interest for practical use. We can make approximations by considering only $1 \leq m \leq M$. The terms $\sum_{l=1}^{n} \frac{\partial \Phi_{m}}{\partial x_{l}}(b)$ are $\neq 0$ since the sensor is supposed $G$-strategic. +

Let us the system (1) and the output function $z(t)=y(b, t) \quad b \in \Omega \quad t \in[0, T]$

The purpose is to reconstruct the unknown gradient initial $\left(y_{1}^{\prime 0}, y_{1}^{\prime 1}\right)$ using the output function (19). The couple $\left.\left(y_{1}^{\prime 0}, y_{1}^{\prime 1}\right)\right)$ is given by the formulae $(17),(18)$. We consider a truncation up to the order $M$, then we obtain

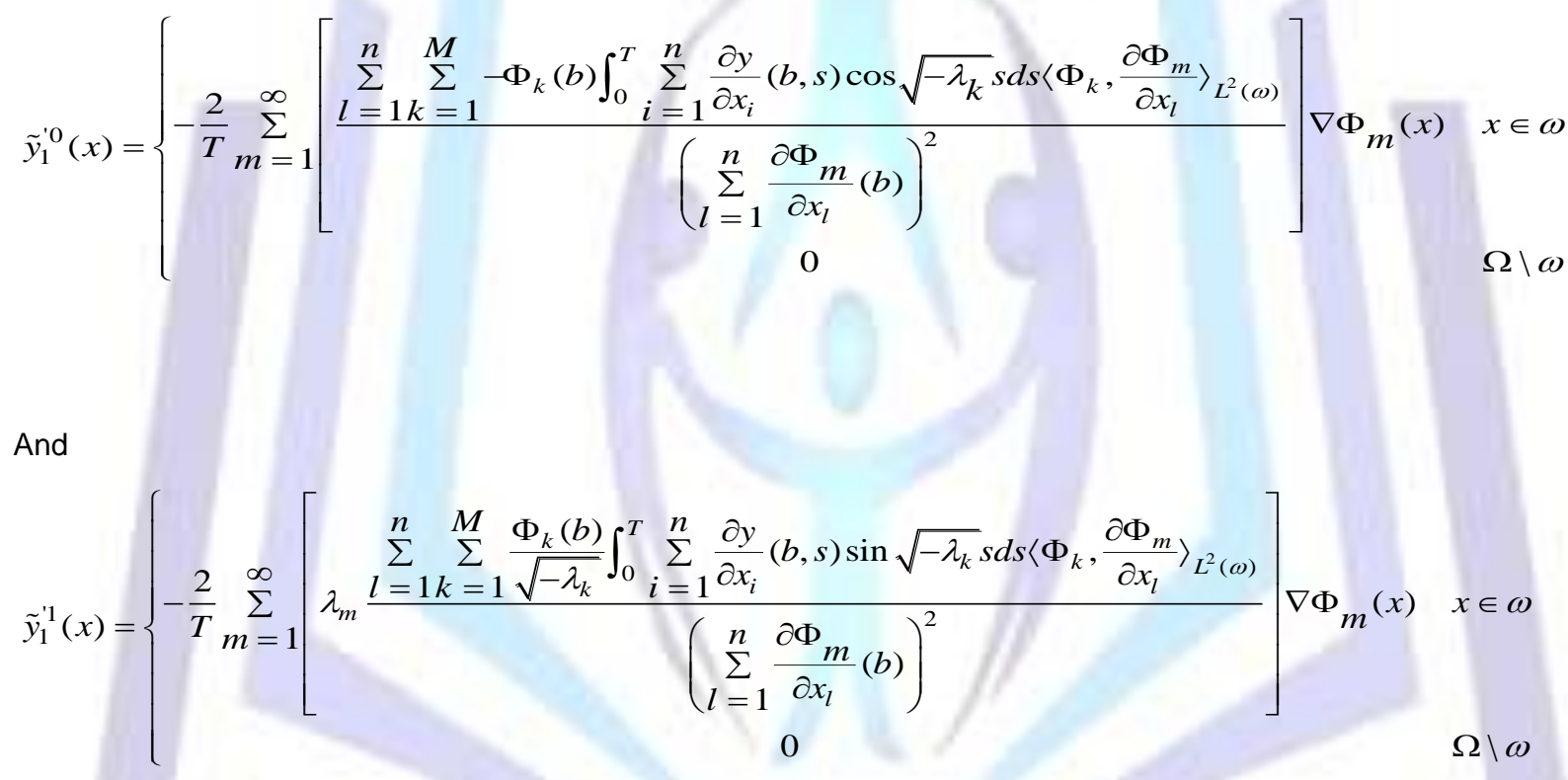

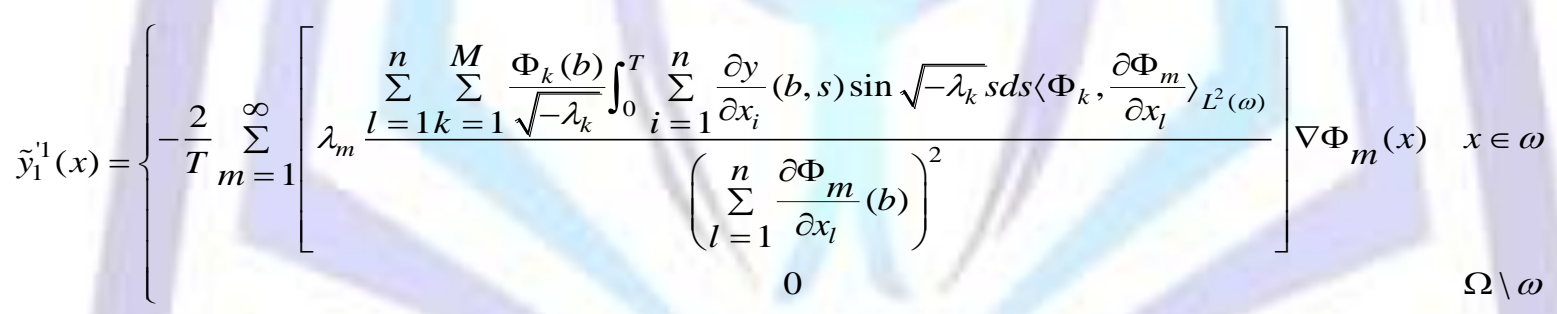

We define a final error (depending on the choice of the sensor location $(b)$ ) by considering

$$
\mathrm{E}^{2}=\square y^{\prime 0}-\tilde{y}_{1}^{\prime 0} \square_{L^{2}(\omega)}^{2}+\square y^{\prime 1}-\tilde{y}_{1}^{\prime 1} \square_{L^{2}(\omega)}^{2}
$$

The good choice of $M$ will be such that $\mathrm{E} \leq \varepsilon$ where $\varepsilon$ is precision test assumed to be small enough.

The general algorithm for computing $\tilde{y}_{1}^{\prime 0}$ and $\tilde{y}_{1}^{\prime 1}$ is the following :

\section{Algorithm:}

1. Choice of the sensor location $b$ and $\varepsilon$.

2. Repeat

$>\quad$ Choice of the approximation order $M$.

$>\quad$ Computation of $y^{\prime 0}$ and $\tilde{y}_{1}^{\prime 0}$ by the formulae ((20) and (21)).

$>\quad M \leftarrow M+1$

Until $\mathrm{E} \leq \varepsilon$. 
3. The estimated initial conditions $\tilde{y}_{1}^{\prime 0}$ and $\tilde{y}_{1}^{\prime 1}$ corresponds to the initial gradient to be observed in the subregion $\omega$.

\section{SIMULATIONS-EXAMPLE}

In this section we present a numerical example, the results are related to the choice of the subregion and the gradient to be observed. Consider the system.

$$
\begin{cases}\frac{\partial^{2} y}{\partial t^{2}}(x, t) \quad= & \left.0.01 \frac{\partial^{2} y}{\partial x^{2}}(x, t) \quad \text { in }\right] 0,1[\times] 0, T[ \\ y(x, 0)=y^{0}(x) \quad, & \left.\frac{\partial y}{\partial t}(x, 0)=y^{1}(x) \text { in }\right] 0,1[ \\ y(0, \mathrm{t})=\mathrm{y}(1, \mathrm{t})=0 & \text { on }] 0, \mathrm{~T}[\end{cases}
$$

Let's consider $\omega=[0.20,0.80]$ and $y^{\prime 0}(x)=\alpha\left(2 x^{3}-3 x^{2}+x\right)$ and $y^{\prime 1}(x)=\beta x(1-x)(2 x-1)$ be the initial gradient to be observed in $\omega$.

For numerical considerations, $\alpha$ and $\beta$ are chosen in order to produce a reasonable amplitude for $y_{1}^{\prime 0}$ and $y_{1}^{\prime 1}$. Here the output is given by means of a pointwise sensor $z(t)=y(b, t)$ with $b=0.54$ and $T=6$, then we obtain the following figures.

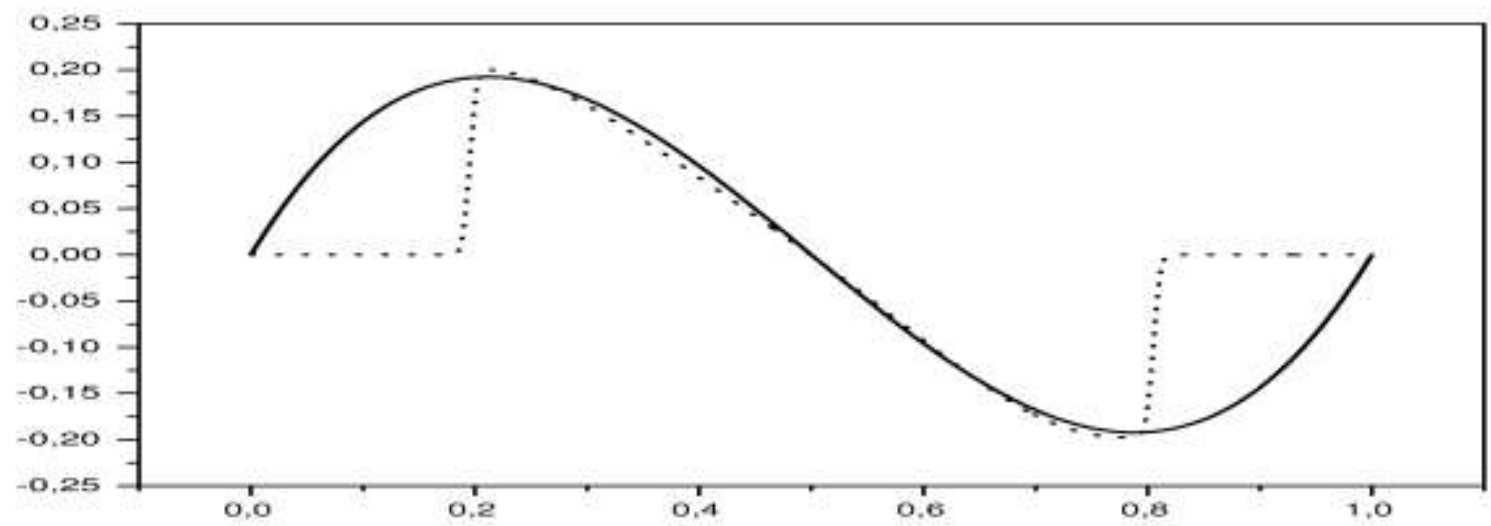

Fig 7 : The exact (continuous line) and estimated (dashed line) state gradient In $\omega$.

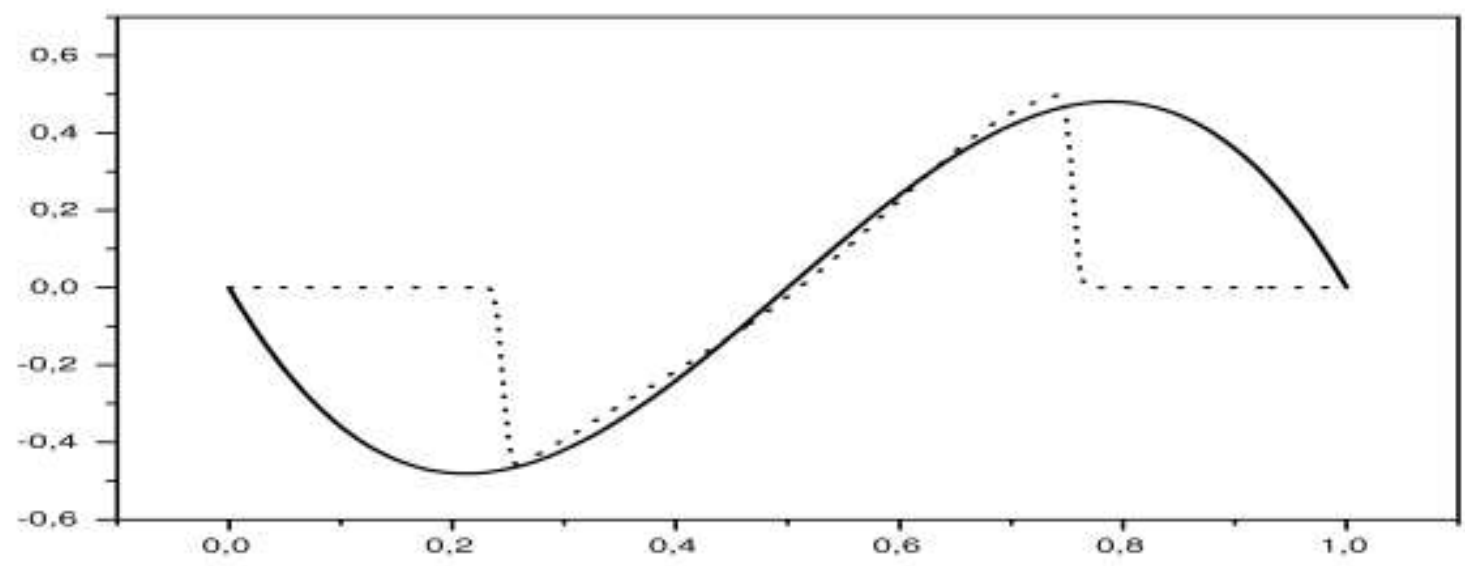

Fig 8 : The exact (continuous line) and estimated (dashed line) speed gradient in $\omega$. 
The reconstruction is obtanied with error equals to: $\mathrm{E}=1.72 \times 10^{-2}$.

For $\omega=\Omega$ we have the following results :

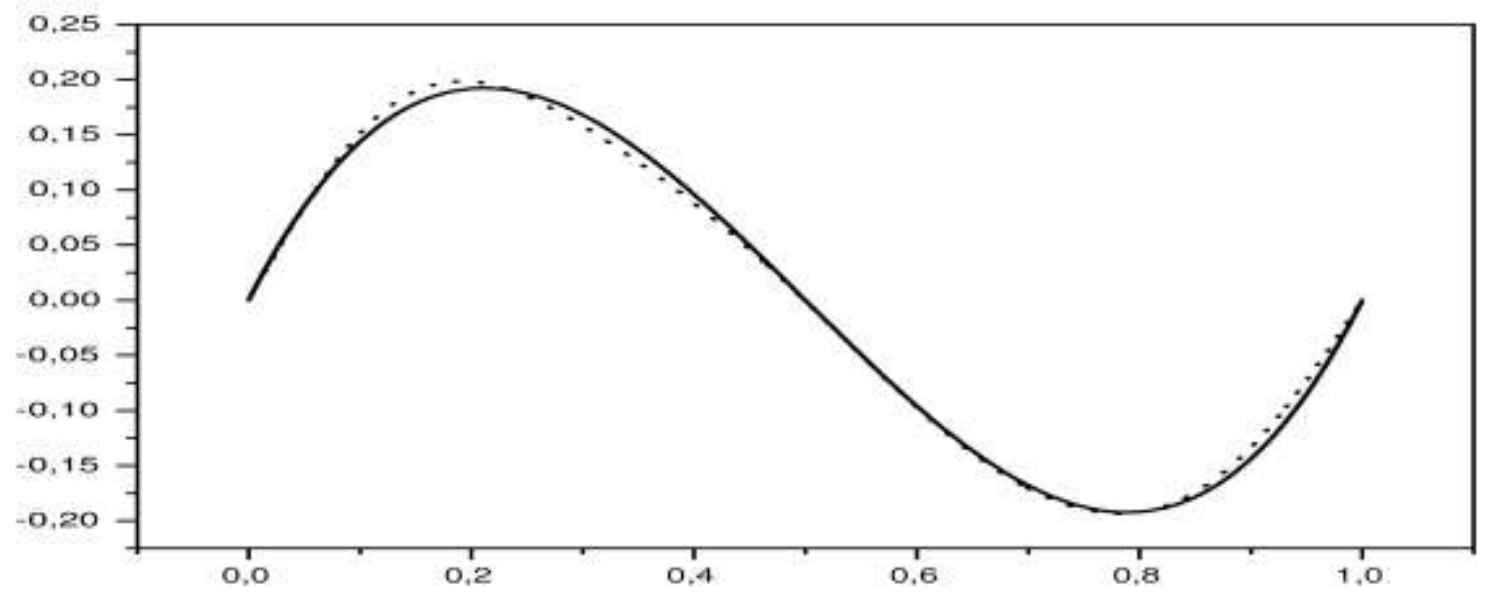

Fig 9 : The exact (continuous line) and estimated (dashed line) state gradient in $\omega$.

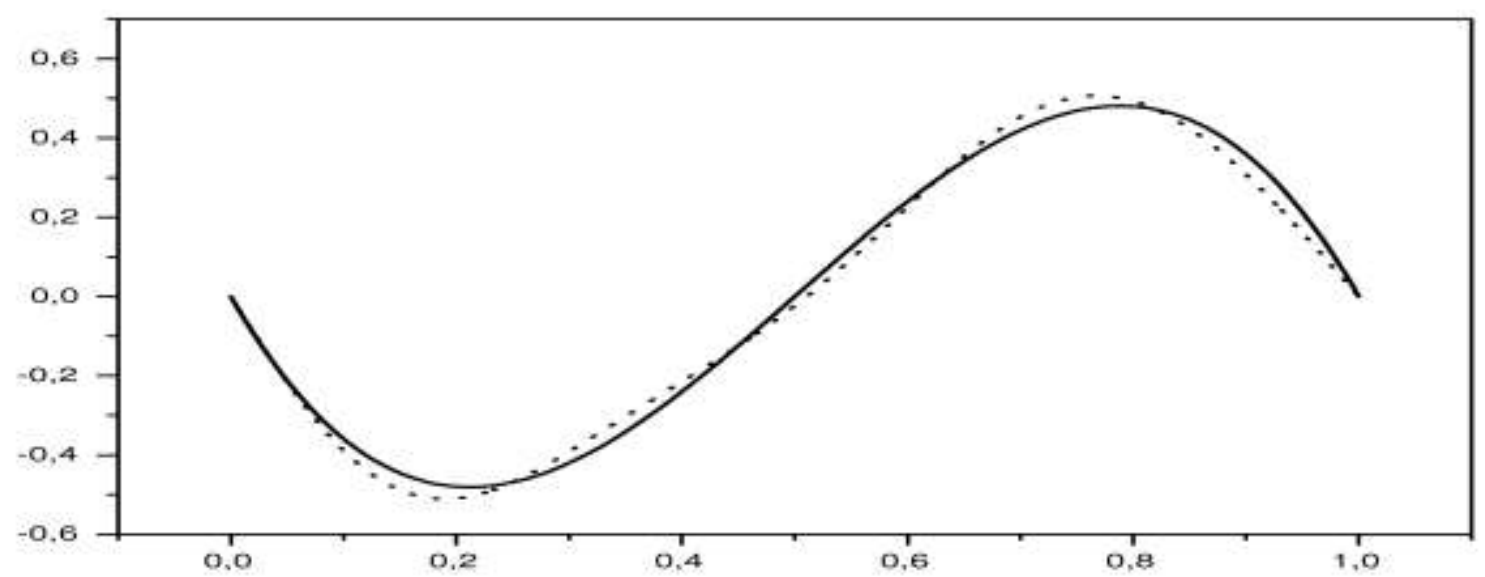

Fig 10 : The exact (continuous line) and estimated (dashed line) speed gradient in $\omega$.

\subsection{Reconstruction error- subregion area}

Here we study numerically the dependence of the gradient reconstruction error with respect to the subregion area of $\omega$, we have the following table.

Table 1 : The reconstruction error with respect to the subregion area.

\begin{tabular}{|c|c|}
\hline The subregion & Reconstruction error \\
\hline$] 0,1[$ & $4.8672 \times 10^{-2}$ \\
\hline$] 0.10,0.90[$ & $3.7339 \times 10^{-2}$ \\
\hline$] 0.15,0.85[$ & $2.6591 \times 10^{-2}$ \\
\hline$] 0.25,0.85[$ & $1.8642 \times 10^{-2}$ \\
\hline$] 0.20,0.80[$ & $1.7255 \times 10^{-2}$ \\
\hline$] 0.24,0.78[$ & $1.2939 \times 10^{-2}$ \\
\hline
\end{tabular}


From Table1, we note that the reconstruction error and the subregion area increase or decrease. This means that the larger the subregion error is the greater the error is. The $G$-observability is realized by means of one pointwise sensor located at $b=0.54$. The results are similar for other types of sensors.

\section{CONCLUSION}

In this work, we studied the notion of regional gradient observability of hyperbolic systems in connection with the sensors parameters. The obtained results are applied to the wave equation in one and two dimensional cases and which give characterization of sensors location. The obtained results are illustrated through numerical example and simulations and can be extended to the boundary case.

\section{ACKNOWLEDGMENTS}

Our thanks to the experts who have contributed towards development of the template.

\section{REFERENCES}

[1] Curtain, R.F. Zwart, H. 1995. An Introduction to Infinite Dimensional Linear Systems Theory, Texts in Applied Mathematics, vol. 21, Springer, New York.

[2] El Jai, A. Simon, M.C. Zerrik, E. 1993. Regional observability and sensor structures, Int. J. Sensors and Actuators, 39 (2), 95-102, (1993).

[3] El Jai, A. and El Yacoubi, S. 1993. On the number of actuators in parabolic systems, Int. J. Appl. Math. Comput. Sci. vol.3, 673-686, (1993).

[4] El Jai, A. and Pritchard, A. J. 1988. Sensors and Actuators in Distributed Systems Analysis, Ellis Horwood series in Applied Mathematics, J. Wiley.

[5] Lions, J. L. and Magenes, E. 1968.Problémes aux limites non homogènes et applications, vol. 1, Dunod, Paris.

[6] Zerrik, E. Bourray, H. 2003. Gradient observability for difusion system, Int. J. Appl. Math. Comput. Sci. 13, 139-150, (2003).

[7] Zerrik, E. Bourray, H. and Boutoulout, A. 2002. Regional boundary observability, numerical approach, International Journal of Applied Mathematics and Computer Science 12(2): 143-151, (2002).

[8] Zerrik, E. Bourray, H. and Boutoulout, A. 2001. Boundary strategic actuators, Sensors and Actuators A 94, 197-203, (2001).

[9] Zerrik, E. Bourray, H. 2003. Flux reconstruction: sensors and simulations, Sensors and Actuators A 109, 34-46, (2003).

[10] Zerrik, E. Bourray, H. and Benhadid, S. 2007. Sensors and Regional observability of the wave equation. Sensor and Actuator journal, A 138, 313-328, (2007).

[11] Zerrik, E. Badraoui, L. 2000. Sensor characterization for regional boundary observability, Int. J. Appl. Math. Comput. Sci. 10 (2), 345-356, (2000).

\section{Author' biography with Photo}

A. Boutoulout is a professor at the University Moulay Ismail of Meknes in Morocco. He obtained his Doctorat d'Etat in System Regional Analysis (2000) at University Moulay Ismail. Professor Boutoulout has published many paper in the area of system analysis and control. Now he is the head of the research team STI (System Theory and Informatics) and a director of Master System Theory and Informatics, in department of Mathematics and Informatics of Faculty of Sciences at the University Moulay Ismail, of Meknes in Morocco.

E-mail : boutouloutali@yahoo.fr

H. Bourray is a professor at the University Moulay Ismail of Meknes in Morocco. He is an assistant professor at the same University. He got his Doctorat in Systems Analysis (2002) at the Faculty of Sciences in Meknes. He is a researched at STI Team, MACS Laboratory at the University Moulay Ismail of Meknes in Morocco.

E-mail address: hbourrayh@yahoo.fr

A. Khazari is a researcher, preparing his Doctorat in Applied Mathematics at the University Moulay Ismail, of Meknes in Morocco. His research area is focused on analysis and control of distributed systems and numerical analysis. At present he is a searcher at STI Team, MACS Laboratory at University Moulay Ismail of Meknes Morocco.

E-mail address: adil0974i@gmail.com 